\title{
The unique role of parietal cortex in action observation: Functional organization for communicative and manipulative actions
}

\author{
Burcu A. Urgen a,b,c,*, Guy A. Orban ${ }^{\mathrm{d}}$ \\ a Department of Psychology, Bilkent University, 06800, Bilkent, Ankara, Turkey \\ ${ }^{\mathrm{b}}$ Interdisciplinary Neuroscience Program, Bilkent University, 06800, Bilkent, Ankara, Turkey \\ ${ }^{\mathrm{c}}$ National Magnetic Resonance Research Center (UMRAM) and Aysel Sabuncu Brain Research Center, Bilkent University, 06800, Bilkent, Ankara, Turkey \\ ${ }^{\mathrm{d}}$ Department of Medicine and Surgery, Neuroscience Unit, University of Parma, Italy
}

\section{A B S T R A C T}

Action observation is supported by a network of regions in occipito-temporal, parietal, and premotor cortex in primates. Recent research suggests that the parietal node has regions dedicated to different action classes including manipulation, interpersonal interactions, skin displacement, locomotion, and climbing. The goals of the current study consist of: 1) extending this work with new classes of actions that are communicative and specific to humans, 2) investigating how parietal cortex differs from the occipito-temporal and premotor cortex in representing action classes. Human subjects underwent fMRI scanning while observing three action classes: indirect communication, direct communication, and manipulation, plus two types of control stimuli, static controls which were static frames from the video clips, and dynamic controls consisting of temporally-scrambled optic flow information. Using univariate analysis, MVPA, and representational similarity analysis, our study presents several novel findings. First, we provide further evidence for the anatomical segregation in parietal cortex of different action classes: We have found a new site that is specific for representing human-specific indirect communicative actions in cytoarchitectonic parietal area PFt. Second, we found that the discriminability between action classes was higher in parietal cortex than the other two levels suggesting the coding of action identity information at this level. Finally, our results advocate the use of the control stimuli not just for univariate analysis of complex action videos but also when using multivariate techniques.

\section{Introduction}

Over the last two decades, neurophysiological and neuroimaging studies in human and nonhuman primates have identified a network of brain regions in occipito-temporal, parietal and premotor cortices that are associated with observing others' actions, known as the Action Observation Network (AON) (Cross et al., 2009a; Caspers et al., 2010; Nelissen et al., 2011; Jastorff et al., 2010). One prominent finding that has guided action observation research to date is that it shares some neural resources with action execution including some regions in the parietal and premotor cortex (Rizzolatti and Craighero, 2004). Comparative functional neuroanatomical studies of action execution have demonstrated that the posterior parietal cortex (PPC) is functionally divided into parts for different actions including grasping, reaching, handto-mouth behavior, looking, and protecting the face or body (Kaas and Stepniewska, 2016; Graziono and Aflalo, 2007). These subdivisions are thought to form "intentional maps" generating preliminary plans for movement (Anderson and Buneo, RA 2002). The relative expansion of the PPC at the level of the inferior parietal lobule (IPL, Van Essen and Dierker, 2007) in humans suggests that it may host additional maps devoted to actions specific to humans (Orban, 2016; Kaas and Stepniewska, 2016). One implication of this research, when combined with the findings of the shared common neural resources for action execution and observation is that parietal cortex may be functionally orga- nized (Goodale and Milner, 1992) and divided into functionally distinct regions for different observed actions in action observation.

There is a growing body of research in action observation that supports the hypothesis of an anatomical segregation in parietal cortex for different action classes, including manipulation, interpersonal interaction, skin displacement, locomotion, and climbing (Jastorff et al., 2010; Abdollahi et al., 2013; Ferri et al., 2015). Action class refers to a group of exemplars that share similar sensorimotor transformations and motor goals (Orban et al., 2021). For instance, grasping or pushing an object are exemplars of the manipulation class, including actions aiming to move or deform an object. Similarly, walking and running are exemplars of the locomotion class, including actions aiming to move the body of the actor in space. Note that for observed actions of others, both action class and exemplar are defined as the category levels of observed action identity, which refers to the observable features of an action that distinguish it from other actions (Orban et al., 2021). Observed action exemplars can be characterized as the ordinate level of the observed action identity whereas observed action classes can be characterized as the superordinate level.

The primary aim of the current study is to build upon previous work on observed action classes with two new classes of actions that are communicative in nature, at least one of which is specific to humans (Liebal and Call, 2012; Graham et al., 2018). In particular, we are interested in whether there are parietal sites that are specifically activated

\footnotetext{
* Corresponding author.

E-mail addresses: burcu.urgen@bilkent.edu.tr (B.A. Urgen), guy.orban@kuleuven.be (G.A. Orban).
} 
by communicative actions, and if so where they are localized in the IPL of the human brain. Here, we make a distinction between direct and indirect communicative hand actions. Direct communicative actions are symbolic hand actions (gestures or emblems, Andric et al., 2013) addressed to a conspecific present in the scene such as waving or signing "no". On the other hand, indirect communication actions involve leaving a symbolic trace (shape or message) in a substrate that can be viewed later by a conspecific such as writing a message or drawing a shape on sand. The common aspect of these two classes is that both have the goal of communicating a message to a conspecific. For direct communication actions, it is an immediate and observable goal, whereas for indirect communications it is a distant goal which is not immediately observable. The observable goal of indirect communication actions is to modify a substrate (e.g. deforming sand by writing on it), which is similar to manipulation actions, whose goal is to modify or move an object. In other words, indirect communication actions and manipulation actions share the immediate goal of modifying an object or a substrate and are very similar in terms of sensorimotor properties (the movements of the effector to achieve a motor goal). However, indirect communication actions differ from manipulations by requiring the actor to have a mental image of the thing they will create (e.g. a message to be written or a shape to be drawn).

A second aim of the current study is to investigate how the three anatomical levels of the AON differ from one another in representing action classes. Computational modeling (Giese and Poggio, 2003) and single cell studies in monkeys ( $\mathrm{J}$ Vangeneugden et al. 2009) suggest that form and motion, both components of an observed action, are processed in the ventral and dorsal pathways of the visual system respectively, and that this information is sent to the occipito-temporal cortex, i.e. the first anatomical level of the AON, to be integrated. This visual information is passed on to the other two levels of the AON, parietal and premotor cortex for further processing (Nelissen et al., 2011). What kind of computations are being carried out in these levels and how they differ from each other is not well understood (Cross et al., 2009b, Wurm and Lingnau, 2015; Chan and Baker, 2015; Urgen et al., 2019). The present study also aims to shed light on this issue with a specific focus on how observed action classes, hence action identity, are represented at the three levels of the AON. By definition, action identity involves two components: 1) body movements of the actor, 2) the changes they produce in the environment, whether it is an object, conspecific, or a substrate (Orban et al., 2021), i.e. the goal of the action. We hypothesize that parietal level of the AON would be the ideal level to represent action identity for several reasons. First, previous research shows anatomical segregation at the level of PPC for representation of different observed action classes (Abdollahi et al., 2013; Ferri et al., 2015; Corbo and Orban, 2017). Second, earlier research shows the role of parietal cortex in representing goals of actions (Anderson and Buneo, RA 2002). Third, there is mounting evidence that occipito-temporal areas visually process all body movements (Beauchamps et al., 2002, Van Geneugden et al., 2009, Jastorff and Orban, 2009; Jastorff et al., 2012) regardless of the action, i.e. no anatomical segregation at this level (Abdollahi et al., 2013; Ferri et al., 2015; Corbo and Orban, 2017). Finally, premotor level is shown to be more sensitive to the effector characteristics of observed actions (Fijii et al., 2008; Jastorff et al., 2010; Di Dio et al. 2013; Fabbri et al., 2016). Thus, we predict that observed action identity is decoded best at the parietal level of the AON.

To this end, we used both univariate and multivariate techniques in a complementary fashion. Using a whole-brain approach and univariate analysis, we functionally localized the regions activated by three different classes of actions as well as the common and specific sites for each action class (Abdollahi et al., 2013). Next, using multivariate pattern analysis (MVPA) (Pereira et al., 2009) we sought to identify which levels of the AON could discriminate between the action classes, and how they differed. Finally, using representational similarity analysis (RSA) (Kriegeskorte et al., 2008; Liu et al., 2013), we compared the neural representational distances between the action classes and their exem- plars across ROIs to reveal the functional properties at each level of the AON.

\section{Methods}

\subsection{Participants}

32 subjects ( 15 females, 17 males, mean age $=26$, $S D=2.87$ ) participated in the study. All participants were right-handed, had normal or corrected-to-normal visual acuity and no history of mental illness or neurological diseases. The study was approved by the Ethical Committee of Parma Province and all volunteers gave written informed consent in accordance with the Helsinki Declaration prior to the experiment.

\subsection{Stimuli}

The stimuli of the experiment consisted of short video clips ( $448 \times 336,50 \mathrm{fps}, 2.6 \mathrm{~s})$ showing an actor from the left side while performing three classes of actions: (1) Indirect communication (Fig. 1A), (2) Direct communication (Fig. 1B), and (3) Manipulation (Fig. 1C). All actions included finger or hand movements. Each action class included four exemplars, and each exemplar had four variants, yielding 16 different video clips per action class. The exemplars of the indirect communication class included writing with the index finger, drawing with the index finger, erasing with the right hand, and sculpting with both hands. All of these were performed on two different types of substrate, sand or dough, by a male or a female actor (which made up the four variants for a given exemplar) to communicate a message (write 'no', draw a heart, erase 'Ciao' ('Hello' in Italian), or sculpt a heart). The exemplars of the direct communication class included waving with the right hand, clapping with both hands, displaying "no" sign by moving the index finger left and right continuously, and displaying "right here" by moving the index finger up and down continuously. Each of these actions was performed by a male or a female actor, and was targeted toward a male or female actor (which made up the four variants for a given exemplar). The exemplars of the manipulation class included grasping an object with the right hand, dropping an object with both hands, dragging an object toward the body with the index finger, and pushing an object away from the body with the index finger. The exemplars are the same as in previous work (Ferri et al., 2015), but the effectors were slightly changed to match those of communicative actions. Each of the manipulative actions were targeted toward a small or a large object, and performed by a male or female actor (which made up the four variants for a given exemplar). We matched the action classes to each other as much as possible (see Table 1 for stimulus properties) by including the same two actors with the same clothing, a target for the action, and equal number of actions performed by a single finger, single hand (unimanual) or two hands (bimanual), and same number of elements in the scene. To leverage the fact that some regions of interests (e.g. parietal cortex) are independent of the effector (Jastorff et al., 2010), we deliberately instructed the actors in the videos to use different effectors but matched them across action classes. Also, by definition, direct communication actions require a conspecific (a second person) to be visible in the videos. To match this large object in the other action class videos, we put a floor lamp in a similar position in the manipulation and indirect communication videos. As explained below, we included additional control conditions to compare the effects of these additional objects in the videos.

In addition to the action stimuli (48 videos in total), two different control stimuli were used: static controls (SC), which consisted of the first, middle, and last frames of each action video, and dynamic controls (DC), which consisted of the optical flow extracted from each action video, superimposed onto a noise pattern, temporally de-correlated between squares of a superimposed grid and reduced to average translation within a square (similar to Ferri et al., 2015). The rationale was 

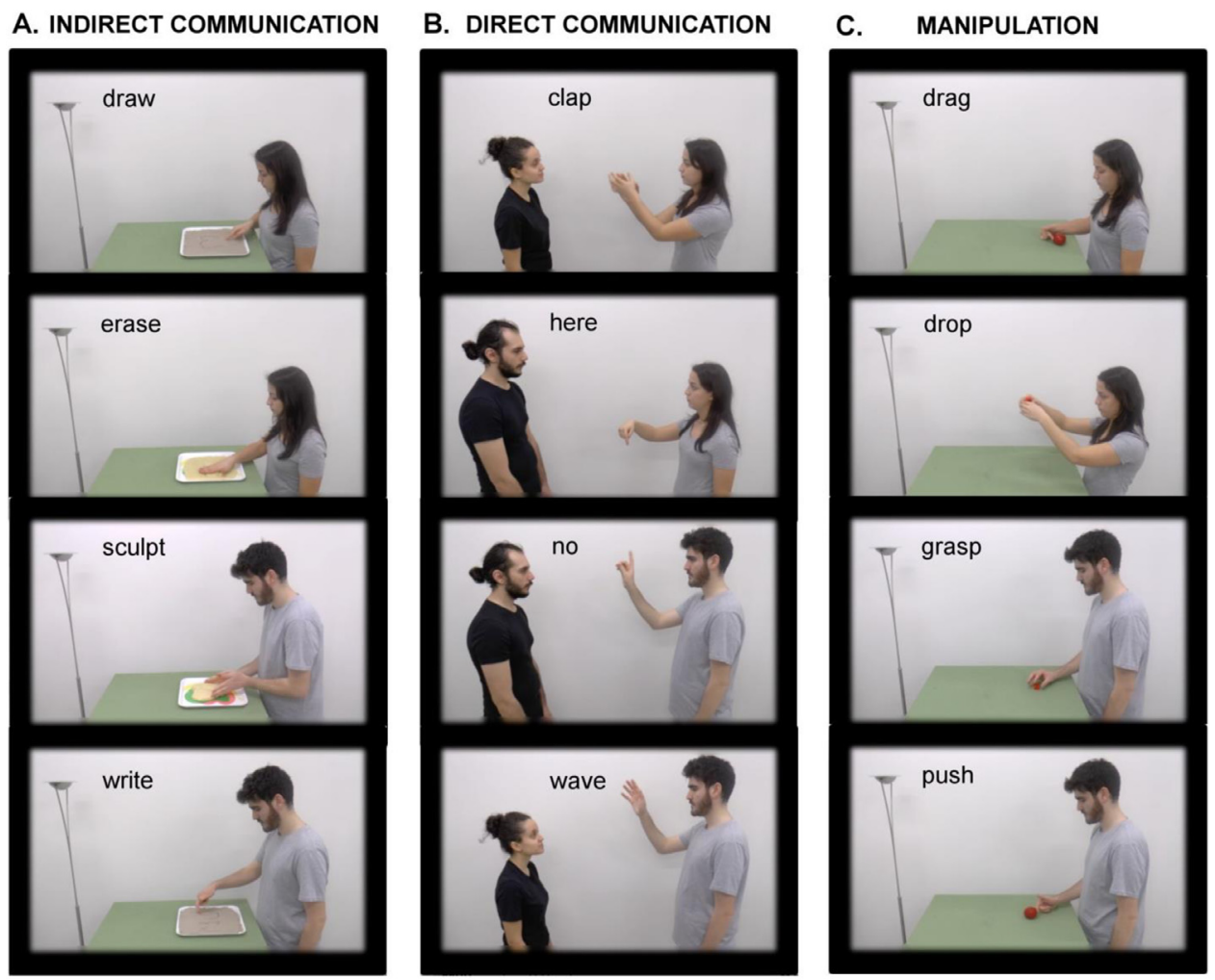

Fig. 1. Static frames from the videos of the three action classes and their four exemplars in the experiment. (A) Indirect communication, (B) Direct communication, (C) Manipulation.

Table 1

Schematic view of the properties of the stimuli for each action class. Each action class included two types of actor (male/female) and two types of target (a substrate for the indirect communication, a person for the direct communication, and an object for manipulation), and was performed manually or bimanually using single or multiple fingers. The number of exemplars that were performed manually and bimanually were equated between action classes.

\begin{tabular}{lllll}
\hline ACTION CLASS/EXEMPLARS & ACTOR & TARGET & MANUAL/BIMANUAL & FINGERS \\
\hline INDIRECT COMMUNICATION & & & & \\
Write & Male/Female & Sand/Dough & Manual & Single \\
Draw & Male/Female & Sand/Dough & Manual & Single \\
Erase & Male/Female & Sand/Dough & Manual & All five \\
Sculpt & Male/Female & Sand/Dough & Bimanual & All ten \\
DIRECT CoMMUNICATION & & & \\
"Here" gesture & Male/Female & Male/Female & Manual & Single \\
"No" gesture & Male/Female & Male/Female & Manual & Single \\
Wave & Male/Female & Male/Female & Manual & All five \\
Clap & Male/Female & Male/Female & Bimanual & All ten \\
MANIPULATION & & & \\
Drag & & & Single \\
Push & Male/Female & Big/Small & Manual & Single \\
Grasp & Male/Female & Big/Small & Manual & All five \\
Drop & Male/Female & Big/Small & Manual & All ten \\
\hline
\end{tabular}

to control for the two components of an observed action: a figural component (shape of the body and scene, including the conspecific), and a motion component (motion vectors of the body). Finally, in all videos or static frames, a blue fixation dot was present near the position where the hand action unfolded. In half of the videos and static frames the fixation dot was slightly above the middle of the effector trajectory, and in the other half, slightly below the effector. Each video or frame was positioned on the screen in such a way that the fixation dot always remained at the center.

\subsection{Experimental design}

The experiment consisted of 10 runs following a block design. In each run, the three action classes ( 3 blocks), and one of the controls (SC or DC) corresponding to the three action classes ( 3 blocks) were presented in a randomized order, and followed by a fixation block lasting as long as the stimulus blocks (41.6 s). This was repeated twice in each run, lasting $582.4 \mathrm{~s}(14 \times 41.6 \mathrm{~s})$. SC and DC were presented in alternating runs. In each SC run, one type of static frame was used (first, middle, or last frame), with their order randomized across SC runs. Within each block 16 videos/images of any given class ( 4 exemplars $\mathrm{x} 4$ variants for a given exemplar) were presented for a total of $41.6 \mathrm{~s}(16 \times 2.6 \mathrm{~s})$. Within a block the 4 exemplars of a given class were presented in a randomized order, but the 4 variants of each exemplar were presented one after the other, defining mini-blocks of exemplars within a given block. The order of the variants was fully randomized as well.

\subsection{Data collection and data preprocessing}

We scanned our subjects at the University Hospital of University of Parma using 3T MR scanner (GE Discovery MR750, Milwaukee, ILL) with an 8-parallel channels receiver coil. Subjects were presented the 
stimuli visually through a head-mounted display (Resonance Technology, Northridge, CA, $60 \mathrm{~Hz}$ refresh rate) with a resolution of $800 \times 600$ pixels while they laid in the scanner, and were asked to fixate a dot at the center of the screen throughout the experiment. The presentation of the stimuli was controlled by E-prime software (Psychology Software Tools, Sharpsburg, PA). In order to reduce head motion during scanning, subjects' heads were supported with cushion pads. During the presentation of the stimuli, eye movements were recorded with an infrared eye tracking system $(60 \mathrm{~Hz}$, Resonance Technology, Northridge, CA).

We acquired functional images during the presentation of the stimuli followed by the acquisition of a high-resolution structural image for anatomical reference. The functional images were acquired using gradient-echoplanar imaging with the following parameters: TR $=3 \mathrm{~s}$, $\mathrm{TE}=30 \mathrm{~ms}$, flip angle $=90,96 \times 96$ matrix with FOV $240(2.5 \times 2.5 \mathrm{~mm}$ in plane resolution), 49 horizontal slices $(2.5 \mathrm{~mm}$ thickness, and $0.25 \mathrm{~mm}$ gap), ASSET factor of 2 . The 49 slices covered the whole brain including cerebellum. In total, $1950 \mathrm{vol}(195 \times 10$ runs) were collected in the experimental session. The structural image was a T1-weighted IRprepared fast SPGR image and covered the whole brain with 186 sagittal slices with $1 \times 1 \times 1 \mathrm{~mm}^{3}$ resolution. Its acquisition parameters were as follows: TE/TR $3.7 / 9.2 \mathrm{~ms}$, inversion time $=650 \mathrm{~ms}$, flip angle $=12$, acceleration factor $($ ARC $)=2$.

\subsection{Data preprocessing and univariate analysis}

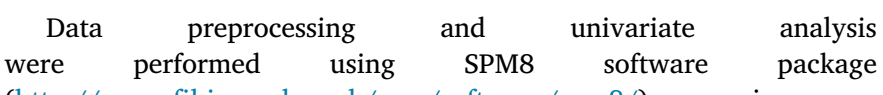
(http://www.fil.ion.ucl.ac.uk/spm/software/spm8/) running under MATLAB (The Mathworks, Natick, MA). The fMRI data of each subject were pre-processed with standard procedures including motion correction, coregistration of the anatomical image and the mean functional image, spatial normalization of all images to a standard stereotaxic space (MNI) with a voxel size of $2 \times 2 \times 2 \mathrm{~mm}$, and smoothing of the resulting images with Gaussian kernel of $6 \mathrm{~mm}$. We discarded 5 subjects who did not fixate well according to the eye movement data (i.e. they made more than 15 saccades per minute for any of the conditions). We thus included 27 subjects (13 females, 14 males) in the final analysis. These subjects averaged 8.4 saccades per minute. Although there was a main effect of condition when we performed a one-way ANOVA on the saccades data considering all experimental conditions and fixation $\left(\mathrm{F}_{6,156}=9.51, p<0.01\right)$, the effect was not driven by the differences between the experimental conditions but rather primarily by the larger number of saccades in the fixation condition (9.7 on average) compared to the experimental conditions, which all averages between 7.2-9.1 saccades, since pair-wise comparisons between the experimental conditions were not significant $(p>0.1)$. We also performed a $3 \times 3$ (action class $\mathrm{x}$ presentation mode) ANOVA on the eye movement data, excluding the fixation condition, and this analysis revealed a main effect of action class $\left(\mathrm{F}_{2,52}=5.15, p=0.009\right)$, driven by more saccades in the manipulation condition compared to indirect communication $(t=-3.21, p=0.007)$, but no effect of presentation mode or interaction. Since the analysis is based on a comparison between presentation modes within classes, and differences between the classes of these differences, our results are unlikely to be explained by differences in fixation.

After preprocessing, we performed a first-level analysis for each subject using a General Linear Model (GLM) with condition onsets and durations. The design matrix consisted of 13 regressors including 7 experimental conditions ( 3 action classes, 3 controls (DC or SC, depending on the run - one for each action class, and 1 fixation), and 6 motion parameters (3 rotations, 3 translations). All regressors were convolved with the canonical hemodynamic response function. Next, we calculated contrast images on the SPM generated in the first level, and then performed a second-level random effects analysis (Holmes and Friston, 1998) for the 27 subjects included in the analysis. We defined three types of statistical maps: Activation map, Specific map, and Common activation map (see below). For these maps, the simple or interaction contrasts were defined at the first level, while conjunctions and masking were made at the second level.

\subsubsection{Whole-brain analysis: activation maps}

We defined various contrasts in order to identify statistical activation maps for each action class (similar to Ferri et al., 2015). Activation maps correspond to the network of brain areas that were significantly more strongly activated by the observation of a particular action class than the observation of the static or dynamic controls of that action class. The activation map for each action class was defined by the contrast [Action $-\left(0.5^{*} \mathrm{DC}+0.5^{*} \mathrm{SC}\right)$ ] and masked inclusively by [Action - Fixation] at $p<0.01$. Please note that DC and SC in each contrast refer to the dynamic and static controls of the respective action. We included in the activation map for each action class those clusters that survived the $p<0.05$ FWE (corrected) at peak level. The activations maps defined in SPM8 were projected onto the flat surface of the left and right hemispheres of the human PALS B12 atlas (Van Essen, 2005) using the Caret software package (Van Essen et al., 2001) since flat maps allow better visualization of sulci and gyri as well as displaying all three views (axial, sagittal, and coronal) of the hemisphere in a single image.

\subsubsection{Whole-brain analysis: specific maps and common activation maps}

We defined specific maps in order to identify the areas that were activated solely by observing a particular action class (similar to Ferri et al., 2015). The specific map for each action class was defined by the conjunction of the two contrasts 1$)$ [(Action $\left.1-\left(0.5^{*} \mathrm{DC}+0.5^{*} \mathrm{SC}\right)\right)-(\mathrm{Ac}-$ tion $\left.\left.2-\left(0.5^{*} \mathrm{DC}+0.5^{*} \mathrm{SC}\right)\right], 2\right)\left[\left(\right.\right.$ Action $\left.1-\left(0.5^{*} \mathrm{DC}+0.5^{*} \mathrm{SC}\right)\right)-($ Action 3 $\left.-\left(0.5^{*} \mathrm{DC}+0.5^{*} \mathrm{SC}\right)\right]$, masked inclusively by the activation map of the action class of interest (Action1) at $p<0.01$, and the contrast [Action1 Fixation] at $p<0.01$, and exclusively by the activation maps of the other two action classes also at $p<0.01$.

We also defined the common activation map across observed action classes, which correspond to the network of brain regions that were significantly activated by all three action classes compared to their static and dynamic controls. As in previous studies (Abdollahi et al., 2013; Ferri et al., 2015), these were defined by the conjunction of three contrasts 1) [Action1 - $\left(0.5^{*} \mathrm{DC}+0.5^{*} \mathrm{SC}\right)$ ], 2) [(Action2 $\left.\left(0.5^{*} \mathrm{DC}+0.5^{*} \mathrm{SC}\right)\right)$ ] , and 3) [Action $3-\left(0.5^{*} \mathrm{DC}+0.5 * \mathrm{SC}\right)$ ] with a conjunction threshold of $p<0.001$ (uncorrected). The specific and common activation maps defined in SPM8 were then projected onto the left and right hemispheres of the human PALS B12 atlas (Van Essen, 2005) using the Caret software package (Van Essen et al., 2001).

\subsubsection{Activity profiles}

We defined the activity profiles as the mean percent signal change in the BOLD response for a given condition compared to the baseline (fixation condition) in a region of interest (ROI). We computed the activity profiles for action classes for the three levels of the Action Observation Network using eight a priori defined ROIs (2 ROIs in occipito-temporal, 5 ROIs in parietal, and $1 \mathrm{ROI}$ in premotor cortex, see the Section 2.6 Identification of ROIs).

Next, we performed an 8 (ROI) x 2 (Hemisphere) x 3 (Action class) $\mathrm{x} 3$ (Presentation mode) ANOVA on the activity profiles for statistical analysis, the four factors being ROI (OTS, MTG, DIPSM, DIPSA, phAIP, PFt, PFcm, Premotor), Hemisphere (Left, Right), Action classes (Indirect communication, Direct communication, Manipulation), and Presentation mode (Video, Static control, Dynamic control).

\subsection{Identification of ROIs}

We aimed to cover all three anatomical levels of the Action Observation Network, namely occipito-temporal, parietal, and premotor levels. To this end, we defined two sets of independent ROIs (Fig. 2) using functional or cytoarchitectonic criteria. The functional ROIs included middle temporal gyrus (MTG) and occipito-temporal sulcus (OTS) in 




Fig. 2. ROIs included in the three levels of the Action Observation Network (occipitotemporal, parietal, and premotor) displayed on surface maps (upper panel) and flat maps (lower panel) for the left (LH) and right hemispheres (RH). Anatomical landmarks are marked in black: CS - Central sulcus, PreCS - Precentral sulcus, PostCS - Postcentral sulcus, IPS - Intraparietal sulcus, POS - Parietooccipital sulcus, STS - Superior temporal culcus, ITS - Inferior temporal sulcus, OTS Occipito-temporal sulcus. ROI abbreviations are as follows: MTG - middle temporal gyrus, OTS - occipito-temporal sulcus, DIPSM - dorsal intraparietal sulcus medial, DIPSA - dorsal intraparietal sulcus anterior, phAIP - putative human AIP.

the occipito-temporal cortex, and premotor cortex (PMC) taken from the maps of Ferri et al. (2015), plus the confidence ellipses for dorsal intraparietal sulcus medial (DIPSM), dorsal intraparietal sulcus anterior (DIPSA), and putative human AIP (phAIP) in the parietal cortex (Jastorff et al., 2010). The phAIP and DIPSA (more precisely its ventral $2 / 3$ ) regions correspond to the rostral motor and caudal visual parts of monkey anterior intraparietal (AIP) area, and DIPSM to anterior part of the lateral intraparietal (LIP) area of the monkey (Orban 2016). These ROIs are known to respond to manipulation actions, which is one of the action classes we used in the present study. The cytoarchitectonic area PFcm in the inferior parietal lobule (Caspers et al., 2006) is included since it has been shown to respond to vocal communication and manipulation actions in previous work (Ferri et al., 2016; Corbo and Orban, 2017). The cytoarchitectonic area PFt (Caspers et al., 2006) is included as well since it anatomically lies between phAIP and PFcm.

\subsection{Multivariate techniques: MVPA and representational similarity analysis}

\subsection{1. $M V P A$}

We performed a 3-way classification between action classes (Indirect Communication, Direct Communication and Manipulation) on the brain activity patterns for each presentation mode, i.e. videos, static controls, and dynamic controls in all ROIs (MTG, OTS, DIPSM, DIPSA, phAIP, PFt, $\mathrm{PFcm}$, and PMC bilaterally).

To prepare the data for classification, we first performed another GLM. In this GLM analysis, each exemplar trial was treated as a different condition regardless of the variants. In other words, the BOLD response was convolved with the mini-blocks instead of the main block (See Section 2.5 Univariate Analysis above). The beta images generated in the first level were fed into the classifier.

In order to perform the classifications, we used support vector machines (SVM) (Cortes and Vapnik, 1995) with a linear basis function and LIBSVM software package (Chang and Lin, 2011). The data were separated into a training set ( $80 \%$ of the total data), and test set $(20 \%$ of the total data), and both were scaled before classification. Five-fold crossvalidation scheme (Pereira et al., 2009) was applied during training.

The prediction accuracy (the ratio of the number of correct predictions to all predictions) was used as the performance metric of the classifier. For the 3-way between-class classifications, the upper limit of above-chance performance with $95 \%$ confidence interval was $41 \%$ in videos, and $44 \%$ in static controls and dynamic controls, computed using the algorithm of Muller-Putz et al. (2008).
After computing the prediction accuracy for each ROI, we performed an 8 (ROI) x 2 (Hemisphere) x 3 (Presentation mode) ANOVA to compare the discriminability of actions across ROIs and presentation modes for 3-way prediction accuracies.

\subsubsection{Representational similarity analysis (RSA)}

In order to study the functional properties of the brain regions involved in action processing, and to investigate the representational space of the brain regions for actions, we used representational similarity analysis (Kriegeskorte et al., 2008). This technique allows investigators to quantify how similar the neural patterns are that correspond to different conditions of an experiment within a certain brain region. In addition, the application of clustering methods (e.g. hierarchical clustering or multidimensional scaling) to the similarity measures allows one to study the representational space of the particular brain region. Further, comparison of the similarity structures across brain regions allows one to study how neural representations evolve along the cortical hierarchy.

We calculated the representational dissimilarity matrix (RDM) in each region of interest (ROI) for each subject by taking the correlation distance ( 1 - correlation) between all pairs for action exemplars using the beta images derived in the first-level (univariate) analysis in SPM8, which resulted in a $12 \times 12$ matrix ( 3 action classes $\mathrm{x} 4$ exemplars for each action class). This was done for the action videos as well as for the static and dynamic controls. For each condition, we computed the grand average dissimilarity matrix by taking the average of all subjects for each ROI. We then applied hierarchical clustering on the grand average RDMs to examine the similarity patterns for action classes, and used multidimensional scaling (MDS) to visualize the geometric distance between action classes. After MDS was applied to the dissimilarity matrices, we computed the average distance of each action class to the other two action classes: $d_{i j}=$ MDS distance between (Action class) ${ }_{i}$ and (Action class) ${ }_{\mathrm{j}}$, and summed them up to compute a distance metric for each ROI. All the steps in RSA were performed using custom scripts in MATLAB.

\section{Results}

\subsection{Univariate analysis}

\subsubsection{Activation maps for action classes}

The first step in the univariate analysis was to functionally localize regions involved in the observation of the three action classes, indirect communication, direct communication, and manipulation. These 


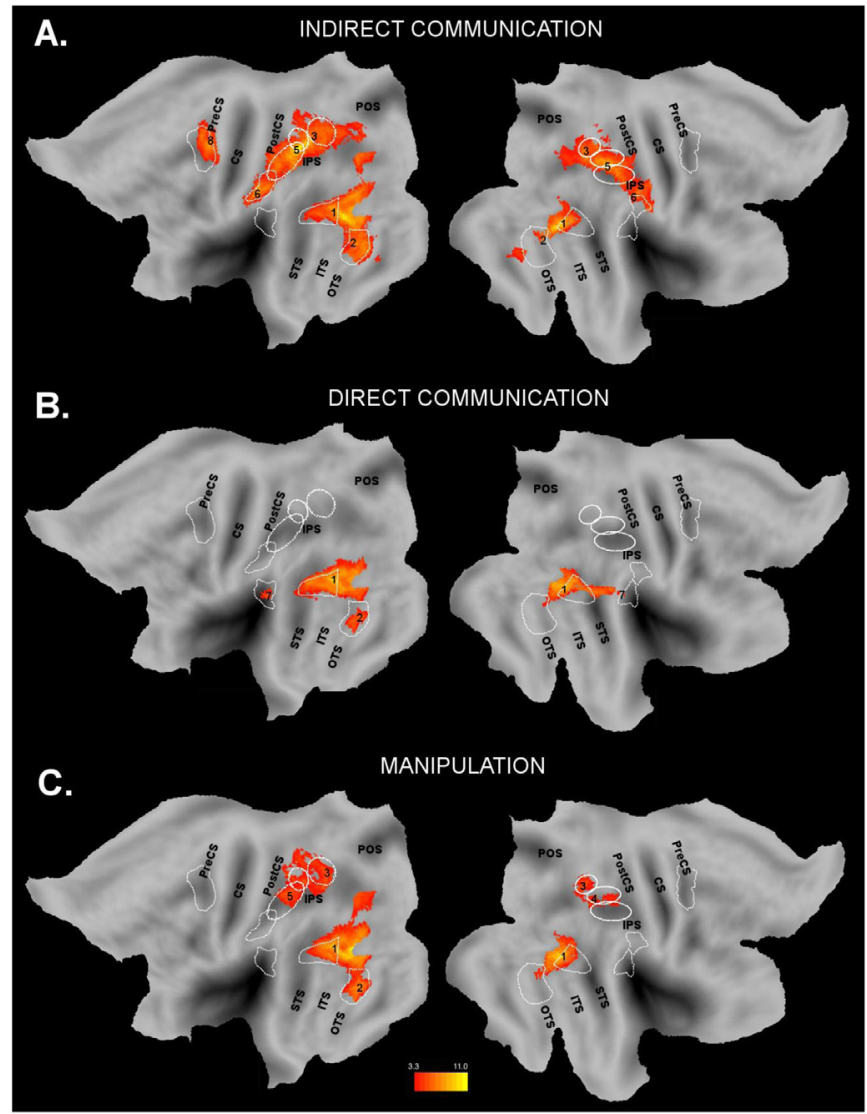

Fig. 3. Activation maps for each action class. The white lines indicate the borders of the ROIs included in the study as shown in Fig. 2. The anatomical landmarks are marked as in Fig. 2. The activation map for each action class was defined by the contrast [Action $-\left(0.5^{*} \mathrm{DC}+0.5^{*} \mathrm{SC}\right)$ ] and masked inclusively by [Action - Fixation] at $p<0.01$, where DC and SC in each contrast refer to the dynamic and static controls of the respective action. (A) Activation map for Indirect communication ( $p<0.05 \mathrm{FWE}$ corrected at peak level). Local maxima were labeled with numbers whose coordinates were listed in Table 2. Color bar shows the t-values. (B) Activation map for Direct communication $(p<0.05 \mathrm{FWE}$ corrected at peak level). Local maxima were labeled with numbers whose coordinates were listed in Table 2. (C) Activation map for Manipulation $(p<0.05$ FWE corrected at peak level). Local maxima were labeled with numbers whose coordinates were listed in Table 2.

regions were shown in the activation map of each action class (Fig. 3). All three maps were bilateral, but showed a clear bias favoring the left hemisphere.

The activation map for indirect communication included regions at all three levels of the Action Observation Network (Jastorff et al., 2010): MTG and OTS as well as the MT+ bilaterally in the occipito-temporal cortex (OTC); DIPSM, DIPSA, phAIP, PFt bilaterally in parietal cortex, and left premotor cortex (Fig. 3A). Although the activation pattern was bilateral, it was left biased especially at the OTC and PMC levels. The local maxima of the activations in each ROI are labeled with numbers on the flat map, and the corresponding SPM coordinates shown in Table 2 with the corresponding numbers. The local maxima in MTG (bilateral) were located in the posterior portion, near the border with $\mathrm{MT}+$, whereas the local maxima in OTS (bilateral) were in the upper part. The local maxima in DIPSM (bilateral) were near the border with DIPSA, as were those in phAIP (bilateral). For PFt, the local maximum was located in its rostral and caudal parts in the left and right hemisphere, respectively. The local maxima in the left premotor cortex was in the posterior part.

The activation map for direct communication included areas MTG and MT+ bilaterally, and left OTS in the occipito-temporal cortex, and bilateral PFcm in the parietal cortex (Fig. 3B). The local maxima in MTG (bilateral) were in the posterior part near the border with MT+, whereas the local maxima of the other sites were located near their centers (See Table 2 for the coordinates).

The activation map for manipulation included left MTG, OTS and MT+ as well as right MTG and MT+ with a small extension into OTS in the occipito-temporal cortex similar to the maps for indirect communication and direct communication. DIPSM, DIPSA, and phAIP were activated bilaterally in the parietal cortex, but with a clear left bias (Fig. 3C). There was also a small cluster in left premotor cortex after small volume correction (SVC) using premotor activation site of the manipulation map from Ferri et al. (2015) as the a priori ROI (10 $\mathrm{mm}$ sphere). The local maxima of its coordinates are listed in Table 2. The local maxima in MTG (bilateral) were in the posterior part near the border with MT+, whereas the local maximum in the left OTS was in the middle of the site. In the parietal cortex, the local maxima of DIPSM (bilateral) were located in its posterior part, of right DIPSA in the posterior portion near the border with DIPSM, and of left phAIP in the center of its posterior half.

\subsubsection{Specific maps and common activation map}

After identifying the activation maps for each class, the second step in the univariate analysis was to identify the regions that were specifically activated by observing a particular class (specific map), and the regions that were commonly activated by observing the three action classes (common activation map).

The specific map for indirect communication was symmetrical, and included a small cluster near OTS bilaterally, the rostral portion of phAIP bilaterally, the rostral portion of PFt bilaterally, a small cluster in the dorsal portion of left premotor, and two small clusters in the dorsal and ventral portions of the right premotor cortex (Fig. 4A). The coordinates of the local maxima were shown in Table 3 . We did not find any clusters exclusively involved in the observation of direct communication or manipulation actions (i.e. the specific maps for these classes were empty).

Next, we identified the common activation map for all action classes (Fig. 4B). The activation map showed a bias favoring the left hemisphere, as did the activation maps. This map included areas in MTG and MT+ bilaterally and left OTS in occipito-temporal cortex as well as a small cluster in the left visual area V3c (Abdollahi et al., 2014). The local maxima in MTG (bilateral) were located in the posterior portion near the border with MT+, whereas the local maximum in the left OTS was in the middle of the site (See Table 3 for the coordinates). There were three common areas in the parietal cortex: PFcm bilaterally, left DIPSM and left V7 (Abdollahi et al., 2014). In all parietal sites, the local maxima were located near their centers.

By definition, the specific map shows the cortical sites in which action classes are most differentiated, whereas the common activation map shows the sites in which action classes are most similar in terms of brain activity. The common map for all three classes (Fig. 4B) included large activation sites in occipito-temporal cortex but relatively few surface nodes in PPC, while the specific map for indirect communication (Fig. 4A) clearly included more nodes in PPC than in occipitotemporal cortex. Indeed, the occipito-temporal activation sites were relatively similar in all 3 activation maps (Fig. 3), while these maps showed clear differences at the PPC level. In order to quantitatively compare in which of the three levels of the AON actions were most distinct and most similar, we computed the number of voxels that were active for a given threshold $(p<0.001$ and $p<0.01)$ around the local maxima of the SPMs of the specific map and the common activation map for the 3 classes. At both thresholds, voxel counts in the parietal cortex outnumbered the other two levels, particularly significantly in the occipitotemporal level, in the specific map, whereas occipito-temporal cortex outnumbered the other two levels in the common map (Table 4; For activation thresholds at $p<0.001$, LH: $\chi 2(2,2503)=1936.16, p<0.001$; RH: $\chi 2(2,1334)=710.37, p<0.001$; For thresholds at $p<0.01, \mathrm{LH}: \chi^{2}$ $(2,4682)=2491.73, p<0.001$; RH: $\chi 2(2,3749)=1692.33, p<0.001)$. 
Table 2

Local maxima of the SPM activation maps ( $p<0.05 \mathrm{FWE}$ at peak level). ROI abbreviations are as follows: MTG - middle temporal gyrus, OTS - occipito-temporal sulcus, DIPSM - dorsal intraparietal sulcus medial, DIPSA - dorsal intraparietal sulcus anterior, phAIP - putative human AIP.

\begin{tabular}{|c|c|c|c|c|c|c|}
\hline & \multicolumn{2}{|c|}{ Indirect Communication } & \multicolumn{2}{|c|}{ Direct Communication } & \multicolumn{2}{|l|}{ Manipulation } \\
\hline & Left & Right & Left & Right & Left & Right \\
\hline \multicolumn{7}{|c|}{ Occipito-temporal } \\
\hline 1. MTG & $-44-704$ & $46-624$ & $-48-686$ & $46-646$ & $-48-684$ & $46-646$ \\
\hline 2. OTS & $-42-60-10$ & $42-62-12$ & $-39-58-18$ & & $-39-58-19$ & \\
\hline \multicolumn{7}{|l|}{ Parietal } \\
\hline 3. DIPSM & $-30-5852$ & $24-6250$ & & & $-26-6448$ & $22-6353$ \\
\hline 4. DIPSA & & & & & & $29-5548$ \\
\hline 5. phAIP & $-36-4848$ & $32-4846$ & & & $-38-4442$ & \\
\hline 6. PFt & $-56-2732$ & $52-2744$ & & & & \\
\hline 7. $\mathrm{PFcm}$ & & & $-46-4220$ & $54-3624$ & & \\
\hline Premotor & & & & & & \\
\hline $\begin{array}{l}\text { 8. Premotor } \\
\text { (SVC) }\end{array}$ & $-30-1458$ & & & & $-28-1252$ & \\
\hline
\end{tabular}

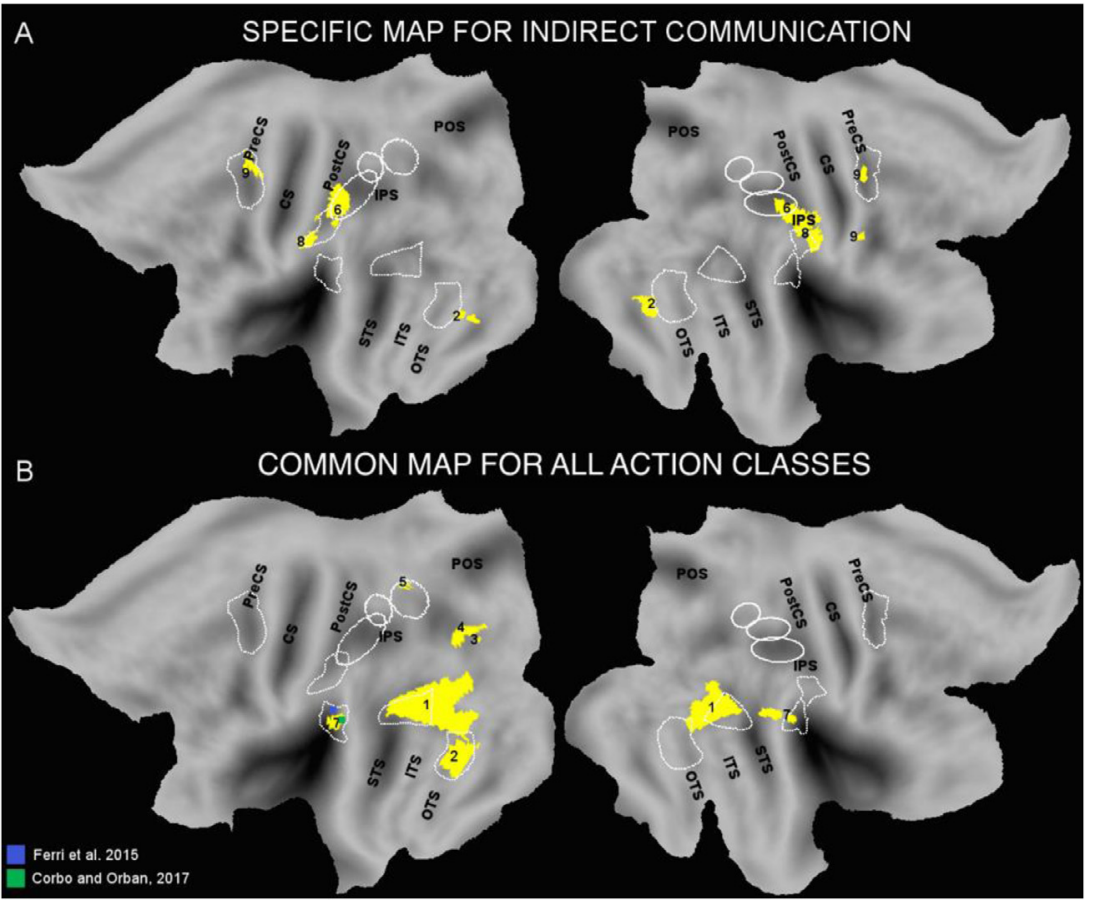

Fig. 4. Specific map and Common activation map. (A) Specific map for Indirect communication $(p<0.001)$. The specific map for Indirect Communication was defined by the conjunction of the two contrasts 1) [(Indirect Communication - $(0.5 * \mathrm{DC}+0.5 * \mathrm{SC}))$ - (Direct Communication - $\left.\left.\left(0.5^{*} \mathrm{DC}+0.5^{*} \mathrm{SC}\right)\right], 2\right)$ [(Indirect Communication $\left.\left(0.5^{*} \mathrm{DC}+0.5^{*} \mathrm{SC}\right)\right)-\left(\right.$ Manipulation - $\left.\left(0.5^{*} \mathrm{DC}+0.5^{*} \mathrm{SC}\right)\right]$, masked inclusively by the activation map of the action class of interest (Indirect Communication) at $p<0.01$, and the contrast [Indirect Communication - Fixation] at $p<0.01$, and exclusively by the activation maps of the other two action classes also at $p<0.01$. (B) Common activation map of all three action classes, Indirect communication, Direct communication, and Manipulation $(p<0.001)$. The common map was defined by the conjunction of three contrasts 1 ) [Indirect Communication - $\left.\left.\left(0.5^{*} \mathrm{DC}+0.5^{*} \mathrm{SC}\right)\right], 2\right)$ [(Direct Communication - $(0.5 * \mathrm{DC}+0.5 * \mathrm{SC}))$ ], and 3) [Manipulation $\left.\left(0.5^{*} \mathrm{DC}+0.5^{*} \mathrm{SC}\right)\right]$ with a conjunction threshold of $p<0.001$ (uncorrected), where DC and SC in each contrast refer to the dynamic and static controls of the respective action. The blue and green dots represent the coordinates from previous studies: observed manipulation actions (Ferri et al., 2015) and observed vocal communication actions (Corbo and Orban 2017). Same ROI and anatomical landmark conventions as in Fig. 2 and Fig. 3.

Table 3

Local maxima of the SPM common activation map and specific map for indirect communication $\left({ }^{*} p<0.001\right.$ uncorrected; ${ }^{* *} p<0.05 \mathrm{FWE}$ at peak level). ROI abbreviations are as follows: MTG - middle temporal gyrus, OTS - occipito-temporal sulcus, DIPSM - dorsal intraparietal sulcus medial, phAIP - putative human AIP.

\begin{tabular}{|c|c|c|c|c|}
\hline & \multicolumn{2}{|c|}{ Specific Map: Indirect Communication } & \multicolumn{2}{|c|}{ Common Map: All 3 Actions } \\
\hline & Left & Right & Left & Right \\
\hline \multicolumn{5}{|c|}{ Occipito-temporal } \\
\hline 1. MTG & & & $-46-684^{* *}$ & $46-644^{* *}$ \\
\hline 2. OTS & $-32-56-20 *$ & $28-50-18 *$ & $-42-60-20 * *$ & \\
\hline 3. V3c & & & $-24-8814 *$ & \\
\hline 4. V7 & & & $-28-8226 *$ & \\
\hline \multicolumn{5}{|l|}{ Parietal } \\
\hline 5. DIPSM & & & $-18-6860 *$ & \\
\hline 6. phAIP & $-44-3648 *$ & $42-3646 *$ & & \\
\hline 7. $\mathrm{PFcm}$ & & & $-48-4022 *$ & $56-3624 *$ \\
\hline 8. PFt & $\begin{array}{l}-54-2436 \\
-58-2646\end{array}$ & $56-1832 *$ & & \\
\hline \multicolumn{5}{|l|}{ Premotor } \\
\hline 9. Premotor & $-30-1458 *$ & $\begin{array}{l}561242 * \\
28-854 *\end{array}$ & & \\
\hline
\end{tabular}


Table 4

Comparison of the three levels of the AON in terms of the number of voxels that are active around the local maxima in the specific map of indirect communication and common map for all three actions with thresholds $p<0.001$ and $p<0.01$.

\begin{tabular}{lllll}
\hline & \multicolumn{2}{l}{$\begin{array}{l}\text { Specific Map for indirect communication } \\
\text { Left }\end{array}$} & Right & \multicolumn{2}{l}{$\begin{array}{l}\text { Common map for all three actions } \\
\text { Left }\end{array}$} & Right \\
\hline$p<0.001$ & & & & \\
Occipito-temporal & 24 & 52 & $\mathbf{1 8 7 4}$ & $\mathbf{7 6 7}$ \\
Parietal & $\mathbf{3 6 2}$ & $\mathbf{3 6 4}$ & 90 & 120 \\
Premotor & 153 & 31 & - & - \\
$\begin{array}{l}p<0.01 \\
\text { Occipito-temporal }\end{array}$ & 142 & 588 & $\mathbf{2 7 1 5}$ & $\mathbf{1 5 5 2}$ \\
Parietal & $\mathbf{9 2 9}$ & $\mathbf{1 2 2 3}$ & 476 & 85 \\
Premotor & 397 & 301 & 23 & - \\
\hline
\end{tabular}

These results highlight that the differentiation of action classes was greatest in the parietal cortex, whereas the commonality was most evident in the occipito-temporal cortex.

\subsubsection{Activity profiles of action classes}

After the whole-brain mapping of regions involved (Section 3.1.1), specifically involved and commonly involved (See Section 3.1.2) in the observation of indirect communication, direct communication, and manipulation actions, the next step in the univariate analysis was to investigate how brain activity for the three action classes differed from one another within the pre-defined regions of interests at the three levels of the AON. For this purpose, we computed the activity profiles of the three action classes (videos) as well as their static and dynamic controls by computing the mean percent signal change in MR signal within each ROI (See Fig. 5 for occipito-temporal and premotor ROIs, and Fig. 6 for parietal ROIs).

Overall, the mean percent signal change for indirect communication was greater than that of direct communication and manipulation for video presentation in all but a few ROIs. On the other hand, the relative activity of direct communication and manipulation varied across ROIs: the activity for direct communication was slightly higher than for manipulation in occipito-temporal areas, whereas it was lower in the parietal areas.

When we compared the activities for the videos with the static and dynamic controls, we found that the activity for video presentation was generally greater than that of the static and dynamic controls. Static controls generally induced stronger activity than did dynamic controls.

Comparing the hemispheres, left hemisphere displays higher activity overall than the right hemisphere, especially for direct communication and manipulation.

In order to examine the differences between conditions statistically, we performed an 8 (ROI) x 2 (Hemisphere) x 3 (Action class) x 3 (Presentation mode) mixed ANOVA including all variables of interest. This omnibus ANOVA showed a main effect of all four factors, ROI, Hemisphere, Action class, and Presentation mode. In addition, the two-way interactions ROI x Presentation mode, Action class x Presentation mode, ROI $x$ Action class, the three-way interactions ROI $x$ Action class $x$ Presentation mode, ROI $x$ Hemisphere $x$ Action class, and the four-way interaction ROI $\mathrm{x}$ Hemisphere $\mathrm{x}$ Action class $\mathrm{x}$ Presentation mode were all significant. (See Table 5 for F-values and p-values of the main effects and the interactions). We corrected for multiple comparisons where appropriate.

Pair-wise comparisons between ROIs showed interesting patterns. Overall, some anatomically nearby regions (e.g. OTS and MTG, DIPSM and DIPSA, PFt and PFcm) did not differ although they differed from the remaining of the ROIs (Figs. 5-6). More specifically, MTG was significantly different from all other ROIs except OTS $(p<0.001)$; OTS was significantly different from all ROIs except MTG $(p<0.001)$; DIPSA was significantly different from all ROIs except DIPSM $(p<0.001)$; DIPSM was significantly different from all ROIs except DIPSA $(p<0.05)$; phAIP was significantly different from all ROIs except Premotor $(p<0.05)$; PFt was significantly different from all ROIs except PFcm $(p<0.05)$; PFcm was significantly different from all ROIs except PFt $(p<0.05)$; and Premotor was significantly different from all ROIs except phAIP $(p<0.05)$. Thus, in general OTC and PMC level differed from the parietal ROIs, which themselves showed some internal differences.

Pair-wise comparisons between the hemispheres showed that activity in the left hemisphere was significantly higher than in the right hemisphere $(p<0.05)$.

On the other hand, pair-wise comparisons between the action classes showed that activity for indirect communication significantly exceeded that of direct communication and manipulation $(p<0.05)$. Direct communication and manipulation did not significantly differ (Figs. 5-6).

When we compared the presentation modes, we found that the video presentation elicited significantly higher activity than the static and dynamic controls $(p<0.01)$. In addition, the static controls were significantly different from the dynamic controls $(p<0.05)$ (Figs. 5-6).

A further important pattern, reflecting the ROI $\mathrm{x}$ action class $\mathrm{x}$ presentation mode interaction, was that the video conditions of the action classes differed more in parietal areas (DIPSM, DIPSA, phAIP, PFt), indirect communication being strongest and direct communication the weakest, compared to the other two levels, occipito-temporal (MTG) and premotor areas (Figs. 5-6). The only exception to this pattern in parietal cortex was PFcm in which the action classes did not differ from each other for the video condition. On the other hand, there were no such differences between the action classes for static controls or dynamic controls.

\subsubsection{Activity profiles of exemplars}

Observing a specific area for indirect communication action class in PFt motivated us to examine the activity profile of the exemplars. Our hypothesis was that if an area is specific for a particular action class, it should differentially respond to its own exemplars, but not to the exemplars of other action classes. On other words, we hypothesized that the activity profile of indirect communication exemplars would be significantly different from each other in PFt, but the activity profile of the manipulation exemplars or direct communication exemplars would not be different from each other in PFt. Furthermore, we hypothesized that this pattern of results would exist only in an activation site which specifically processes a particular action, i.e. PFt in the present study. To this end, we ran in each parietal ROI (PFt, phAIP, PFcm, DIPSM, DIPSA) a one-way ANOVA across exemplars for the three action classes. We corrected for 18 comparisons. Our results showed that the effect of exemplars was significant for indirect communication in PFt bilaterally (left: $\mathrm{F}_{3,78}=21.73, p<0.001$; right: $\mathrm{F}_{3,78}=24.77, p<0.001$ ), and DIPSM $\left(\mathrm{F}_{3,78}=9.41, p<0.001\right)$ and DIPSA $\left(\mathrm{F}_{3,78}=18.36, p<0.001\right)$ on the right. The variation across the exemplars was larger in PFt compared to the other two ROIs (Fig. 7). There was no effect of exemplars of other action classes in none of these ROIs. We also performed a 3 (presentation mode) $x 4$ (exemplar) ANOVA on the activity profiles for each of the four ROIs we found in the previous analysis, to check whether the activity patterns were specific to video conditions (as compared to control condi- 

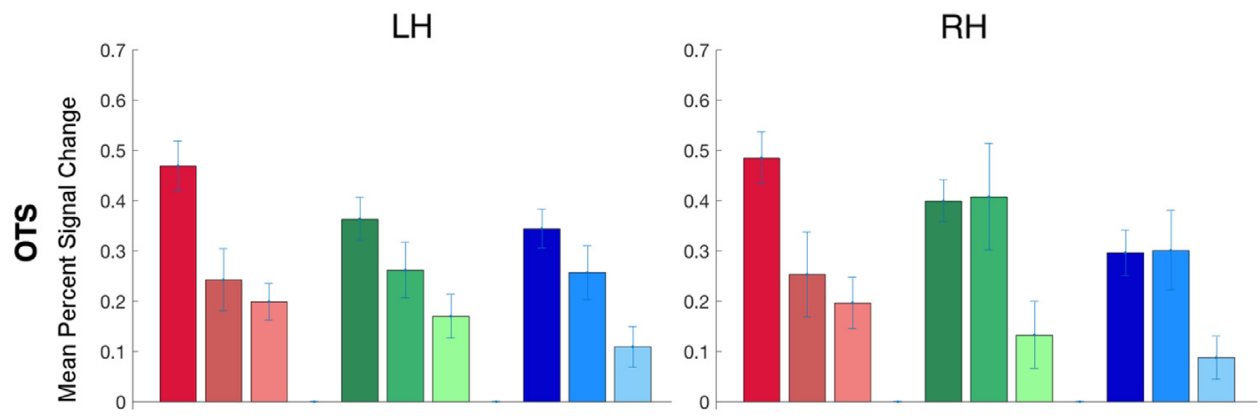

Fig. 5. Activity profiles of the three action classes and their static and dynamic controls in ROIs in the occipito-temporal and premotor cortex. Video refers to the action stimulus. SC Static control, DC - Dynamic control, LH - Left hemisphere, RH - Right hemisphere. Error bars show the standard error. (A) Left OTS and right OTS. (B) Left MTG and right MTG. (C) Left premotor and right premotor. ROI abbreviations are as follows: MTG - middle temporal gyrus, OTS - occipito-temporal sulcus.


Table 5

Main effects and interaction effects of the ANOVA 8 (ROI) x 2 (Hemisphere) $x 3$ (Action class) $\times 3$ (Presentation mode) on univariate brain activity (Significant effects are marked in bold in the first and third column).

\begin{tabular}{|c|c|c|}
\hline & F-value & p-value \\
\hline ROI & $\mathrm{F}_{7,357}=56.64$ & $p<0.001$ \\
\hline Hemisphere & $\mathrm{F}_{1,51}=5.27$ & $p<0.05$ \\
\hline Action class & $\mathrm{F}_{2,102}=6.16$ & $p<0.005$ \\
\hline Presentation mode & $\mathrm{F}_{2,51}=21.95$ & $p<0.001$ \\
\hline ROI $x$ Hemisphere & $\mathrm{F}_{7,357}=1.86$ & $p=0.08$ \\
\hline ROI $x$ Action class & $\mathrm{F}_{14.714}=6.70$ & $p<0.001$ \\
\hline ROI x Presentation Mode & $\mathrm{F}_{14,357}=7.69$ & $p<0.001$ \\
\hline Hemisphere $\mathrm{x}$ Action class & $\mathrm{F}_{2,102}=2.19$ & $p=0.12$ \\
\hline Hemisphere $\mathrm{x}$ Presentation mode & $\mathrm{F}_{2,51}=0.98$ & $p=0.38$ \\
\hline Action class $\mathrm{x}$ Presentation mode & $\mathrm{F}_{4,102}=4.49$ & $p<0.005$ \\
\hline ROI $x$ Hemisphere $x$ Action class & $\mathrm{F}_{14,714}=2.56$ & $p<0.005$ \\
\hline ROI $x$ Action class $x$ Presentation mode & $\mathrm{F}_{28,714}=2.50$ & $p<0.001$ \\
\hline ROI $x$ Hemisphere $x$ Presentation mode & $\mathrm{F}_{14,357}=0.45$ & $p=0.96$ \\
\hline Hemisphere $\mathrm{x}$ Action class $\mathrm{x}$ Presentation mode & $\mathrm{F}_{4,102}=0.75$ & $p=0.56$ \\
\hline ROI $x$ Hemisphere $x$ Action class $x$ Presentation mode & $\mathrm{F}_{28,714}=1.93$ & $p<0.005$ \\
\hline
\end{tabular}

tions). We found a main effect of exemplars in the right PFt $(p=0.013)$, right DIPSM $(p<0.001)$ and right DIPSA $(p<0.001)$; a main effect of presentation mode (left PFt: $p<0.001$; right PFt: $p=0.015$; right DIPSM: $p<0.001$; right DIPSA: $p<0.001$ ) and most importantly, interaction (left PFt: $p<0.001$; right PFt: $p=0.015$; right DIPSM: $p=0.001$; right DIPSA: $p<0.001)$ in all ROIs. Video condition was always larger than the other two control conditions $(p<0.05$; except right PFt, where video differed only from dynamic control $(p=0.023)$ ), although the control conditions did not differ $(p>0.1)$.

\subsection{MVPA: 3-way pattern classification across action classes}

In order to investigate how well the three levels of the AON discriminated the indirect communication, direct communication, and manip- 


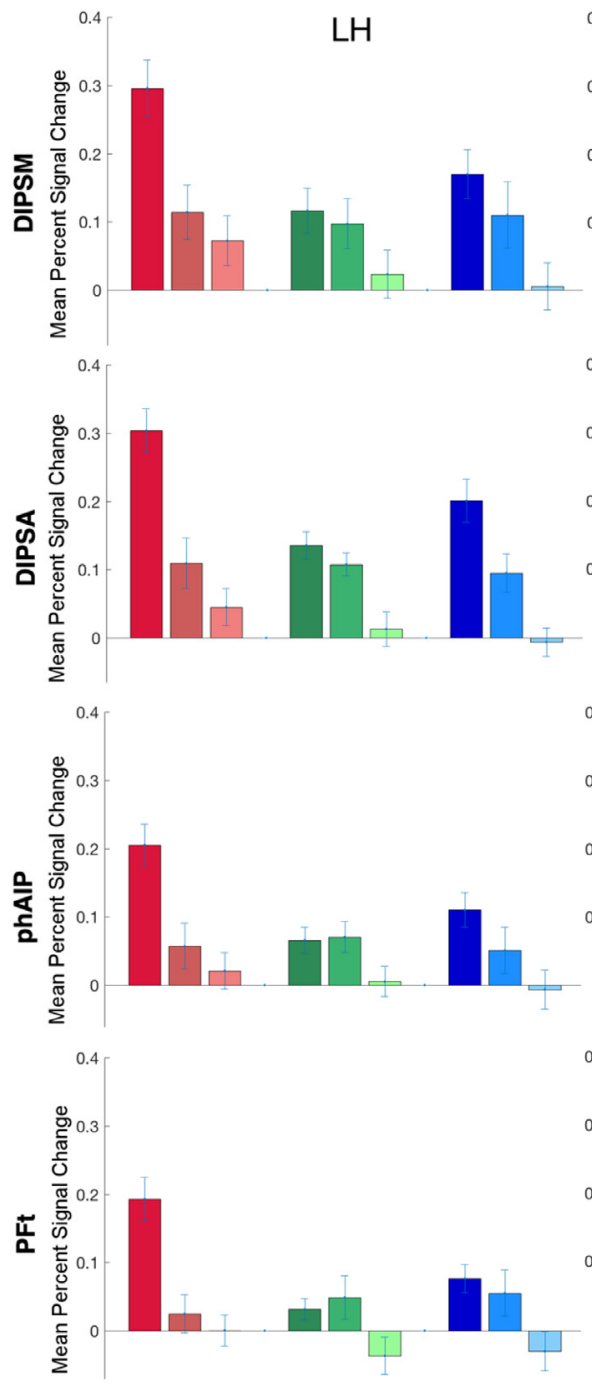

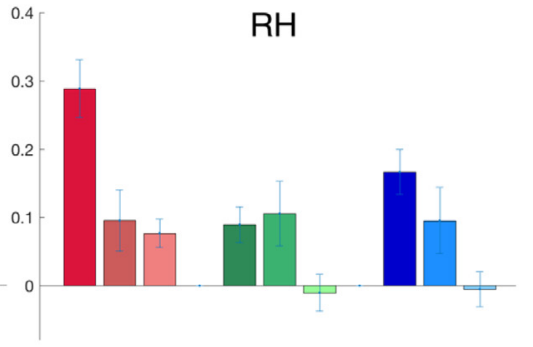

Fig. 6. Activity profiles of the three action classes and their static and dynamic controls in ROIs in the parietal cortex. Video refers to the action stimulus. SC - Static control, DC - Dynamic control, LH - Left hemisphere, RH - Right hemisphere. Error bars show the standard error. (A) Left DIPSM and right DIPSM. (B) Left DIPSA and right DIPSA. (C) Left phAIP and right phAIP. (D) Left PFt and right PFt. (E) Left PFcm and right PFcm. ROI abbreviations are as follows: DIPSM - dorsal intraparietal sulcus medial, DIPSA - dorsal intraparietal sulcus anterior, phAIP - putative human AIP.



ulation actions, we applied a 3-way pattern classification to the neural activity patterns elicited by the video presentation of the three action classes in each ROI. We further investigated using the control conditions, whether the form or motion components of the observed actions could be sufficient to discriminate between the action classes. For this, we performed the 3-way pattern classification to the neural activity patterns both for the static controls (form information) and dynamic controls (motion information) of the three action classes. One should be cautious in interpreting the classification results for each presentation mode on its own, as videos, static frames and even the dynamic control videos are complex stimuli consisting of many components. For example, the static frames do not include the static hand alone but also the actor, a table, and a conspecific or a lamp on the left depending on the action class. Even the dynamic controls include the local motion related to the hand action, but at least for manipulation also the motion of the object resulting from the action.

Overall, the actions were discriminated significantly above chance in all ROIs for videos (chance $41 \%$ ) as well as the static and dynamic controls (chance 44\%, Fig. 8). However, the discrimination patterns for videos and the controls differed. When we compared prediction accuracies between the videos and the controls, we found that parietal areas (including DIPSM, DIPSA, phAIP) showed higher prediction accuracies for videos compared to the static and dynamic controls, whereas occipito-temporal (left OTS) and premotor (left) ROIs showed higher prediction accuracies for the static and dynamic controls compared to videos. An 8 (ROI) x 2 (Hemisphere) x 3 (Presentation mode) ANOVA 

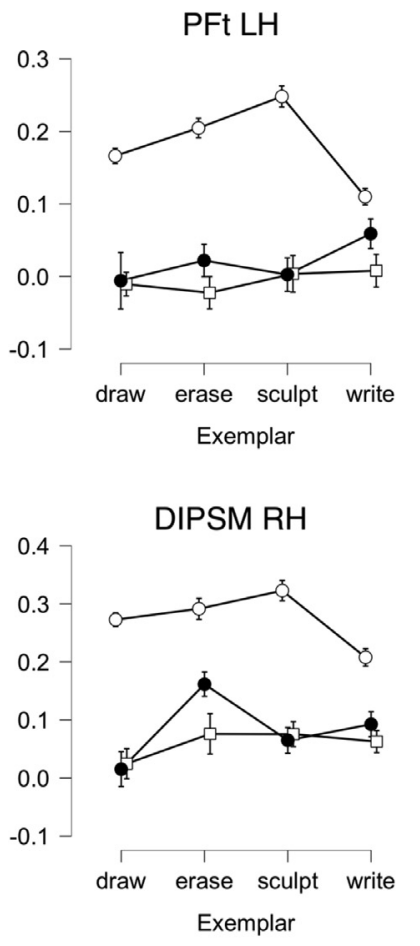

\section{PFt RH}

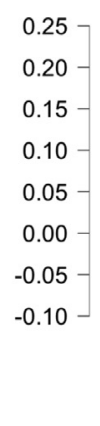

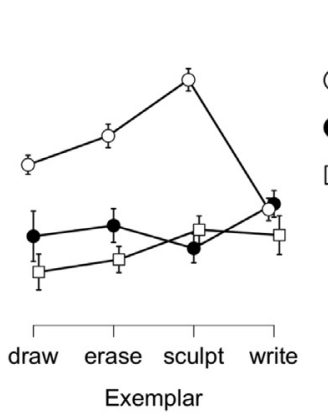

Presentation Mode

$\bigcirc$ Video

Static

Dynamic

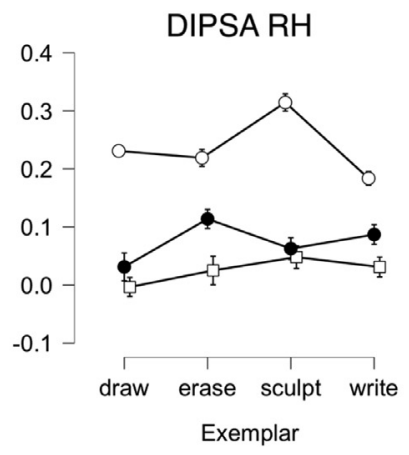

Fig. 7. Activity profiles of the exemplars for all action classes in PFt. LH - Left hemisphere, RH - Right hemisphere. Error bars show the standard error.

$\mathrm{LH}$

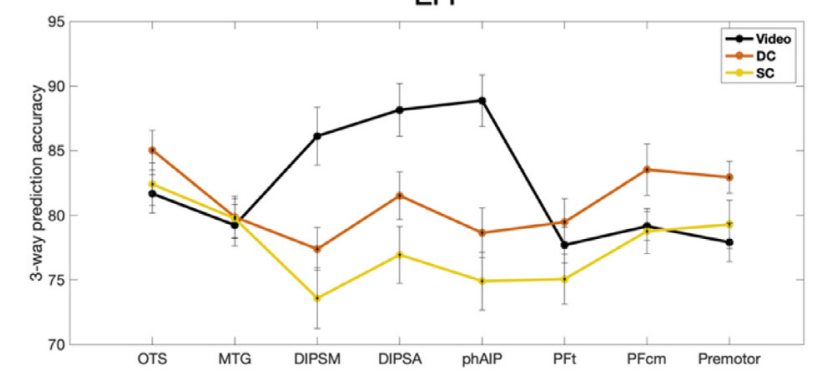

$\mathrm{RH}$

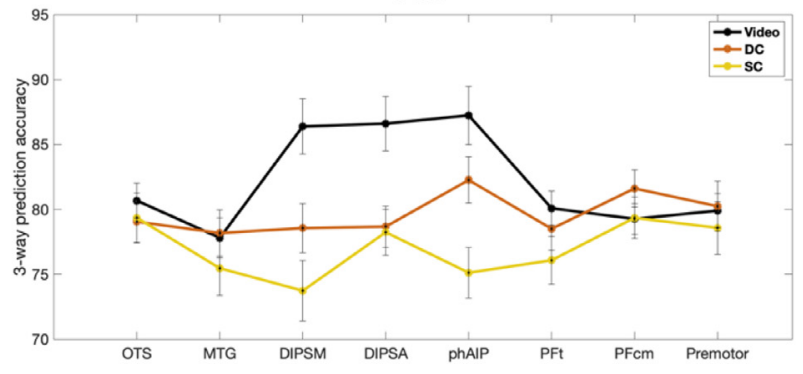

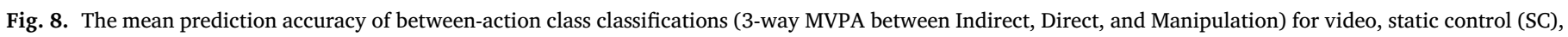

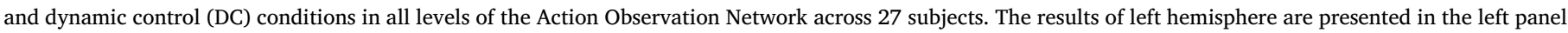

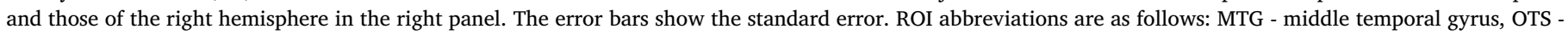
occipito-temporal sulcus, DIPSM - dorsal intraparietal sulcus medial, DIPSA - dorsal intraparietal sulcus anterior, phAIP - putative human AIP.

Table 6

Main effects and interaction effects of the ANOVA 8 (ROI) x 2 (Hemisphere) $\mathrm{x} 3$ (Presentation mode) on 3-way prediction accuracy (MVPA) (Significant effects are marked in bold in the first and third column).

\begin{tabular}{lll}
\hline & F-value & p-value \\
\hline ROI & $\mathrm{F}_{7,182}=3.52$ & $\boldsymbol{p}<\mathbf{0 . 0 1}$ \\
Hemisphere & $\mathrm{F}_{1,26}=2.83$ & $p=0.10$ \\
Presentation mode & $\mathrm{F}_{2,52}=14.78$ & $\boldsymbol{p}<\mathbf{0 . 0 0 1}$ \\
ROI x Hemisphere & $\mathrm{F}_{7,182}=1.30$ & $p=0.25$ \\
ROI x Presentation Mode & $\mathrm{F}_{14,364}=6.97$ & $\boldsymbol{p}<\mathbf{0 . 0 0 1}$ \\
Hemisphere x Presentation Mode & $\mathrm{F}_{2,52}=1.23$ & $p=0.30$ \\
ROI x Hemisphere x Presentation Mode & $\mathrm{F}_{14,364}=0.89$ & $p=0.57$ \\
\hline
\end{tabular}

on the 3-way prediction accuracies showed a main effect of ROI, and a main effect of Presentation mode, as well as an interaction effect of ROI $x$ Presentation mode (See Table 6 for the F-values and p-values). Pairwise comparisons between the presentation modes showed that the action class discrimination with the video presentation was significantly greater than the static control $(p<0.001)$ but not the dynamic control, and that the dynamic control was significantly greater than the static control $(p<0.01)$.

Next, we examined the discrimination with each presentation mode separately to further examine the variability across the three levels of the AON. For videos, the discrimination between action classes was much stronger in parietal cortex than in occipito-temporal and premotor cortex. One-way ANOVA (ROI) of the 3-way prediction accuracy for videos showed a main effect of ROI in both hemispheres (Left: $F(7182)=10.40$, $p<0.001$; Right: $\mathrm{F}(7182)=7.38, p<0.001)$. Pair-wise differences between ROIs showed clear distinctions between the parietal cortex (especially DIPSA and phAIP) and the other two levels in the left hemisphere: DIPSA and phAIP discriminated the action classes significantly better than MTG and Premotor $(p<0.05)$. Furthermore, there were several differences between parietal ROIs as well: DIPSA and phAIP discriminated significantly better than PFt and PFcm $(p<0.01)$.

As expected from the interaction between ROIs and presentation mode, the discrimination between the action classes for the action videos was significantly better than static and dynamic controls in parietal regions (DIPSM: video vs. static control, $t=6.86, p<0.001$; video vs. dynamic control, $t=4.51, p=0.002$; DIPSA: video vs. static control, $t=5.33, p<0.001$; video vs. dynamic control, $t=3.97, p=0.024$; phAIP: 
video vs. static control, $t=7.11, p<0.001$; video vs. dynamic control, $t=4.15, p=0.012$ ). On the other hand, the discrimination between the action classes for static and dynamic controls, unlike the video presentation, was better in occipito-temporal than in parietal cortex: Oneway ANOVA (ROI) of the 3-way prediction accuracy for both controls showed a main effect of ROI only in the left hemisphere (Static controls: $\mathrm{F}(7182)=2.59, p<0.05$; Dynamic control: $\mathrm{F}(7182)=2.47, p<0.05)$, and pair-wise differences showed that for both controls OTS discriminated the action classes significantly better than DIPSM $(p<0.05)$.

\section{3. $R S A$}

In order to investigate the neural representational distances between the three action classes, indirect communication, direct communication, and manipulation, and how they changed across the three levels of the AON, we employed representational similarity analysis. We computed the representational similarity matrix (RSM) for each ROI, and then applied hierarchical clustering and multidimensional scaling (MDS) on the dissimilarity matrices to visualize the distances between action classes and their exemplars for videos, static controls and dynamic controls.

For videos, the RSM for each ROI showed clear distinctions between the three action classes: the neural representations for the exemplars of each class were more similar to each other compared to exemplars of the other two classes. The RSMs of example ROIs from each level of the AON were shown in Fig. 9A: MTG from left occipito-temporal cortex, phAIP from left parietal cortex, and left premotor cortex (other ROIs were not shown due to space limitations). The hierarchical clustering (Fig. 9B) and MDS (Fig. 9C) applied to the dissimilarity matrices (1-similarity matrix) showed this pattern more clearly wherein the exemplars of each action class were more closely clustered to each other compared to the exemplars of the other two action classes. In addition, hierarchical clustering also showed that the overall representational geometry was consistent across the three levels, in which the neural representation for manipulation and direct communication were more similar to each other compared to the indirect communication (See Fig. 9B for the clustering structure in the dendrograms of the example ROIs). The only exception to this pattern was observed in left PFt in which the neural representations for direct and indirect communication were closer to one another compared to manipulation.

Although the neural representational geometry was consistent across the three levels of the AON, the representational distance between the action classes changed across the levels. Overall, the representational distance between the action classes was greatest in the premotor cortex, followed by the parietal cortex and occipito-temporal cortex as evidenced for the example ROIs by the height of the dendrograms in Fig. 9B and geometric total distance in the MDS plots in Fig. 9C.

In order to generalize these observations for the video conditions, and to clearly see the neural distances between each action class pair and how they changed across the three levels of the AON (similar to our approach in pattern classification, see Section 3.2.1.2), we plotted the pairwise MDS distances between action classes, averaging over exemplars within each action class. The results were shown in Fig. 9D (open circles). Overall, there was a quite consistent pattern in which distances between action pairs were greater in the premotor cortex followed by parietal, and by occipito-temporal cortex for videos. A 2 (Hemisphere) x 8 (ROI) ANOVA on the between-class differences confirmed these results. There was a main effect of ROI $(\mathrm{F}(7182)=7.88, p<0.001)$. Posthoc tests revealed that there were significant differences between MTG (occipitotemporal) and all other ROIs (MTG vs. DIPSM: $p=0.04$; MTG vs. DIPSA: $p=0.006$; MTG vs. phAIP: $p=0.001$; MTG vs. PFt: $p=0.000$; MTG vs. PFcm: $p=0.083$ (marginal); MTG vs. Premotor: $p=0.000$ ), as well as differences between OTS and Premotor $(p=0.000)$, and DIPSM and Premotor $(p=0.031)$. All other pair-wise differences were not significant $(p>0.05)$. However, Fig. 9D shows that these changes in distance across ROI were very similar for the two control conditions. Indeed, An omnibus 3-way ANOVA including the presentation mode as a factor as well -2 (Hemisphere) x 8 (ROI) x 3 (Presentation Mode)- showed that there was an effect of $\mathrm{ROI}(\mathrm{F}(7182)=8.05, p<0.001)$ and presentation mode $(\mathrm{F}(2,52)=8.80, p<0.001)$ but no interaction between the two $(\mathrm{F}(14,364)=1.40, p=0.15)$, suggesting that the change across ROIs is similar for the three presentation modes (videos, static controls, and dynamic controls).

\section{Discussion}

Our results indicate that parietal cortex hosts dedicated sites for observing human-specific indirect communication actions. They also show that action classes are most sharply discriminated at the parietal level of the AON, suggesting action identity is coded at this level. Finally, our study also reports some caveats in the use of multivariate techniques, particularly with regard to classification of complex stimuli.

\subsection{Functional organization of the human parietal cortex for observed actions}

The present study contributes to the growing body of literature indicating that observing different classes of actions activates different anatomical regions in human parietal cortex (Abdollahi et al., 2013; Ferri et al., 2015; Corbo and Orban, 2017). Observing indirect communication, a class of uniquely human actions, exclusively activated PFt and anterior phAIP as opposed to manipulation and direct communication. These results suggest that expansion of human IPL during evolution (Van Essen and Dierker, 2007) may have enabled the representation of action classes specific to humans. The specific PFt activation during observation of indirect communication actions may be accounted for by several sensorimotor differences between action classes, which assumes that observing and planning actions of a given class overlap in PPC (Ferri et al., 2015). First of all, there is an overlap between the PFt activation and aSMG, an area involved in observing tool actions (Peeters et al., 2009). Despite the absence of tools, indirect communication actions may have inherent tool-like kinematics (Peeters et al., 2013) since the hand was used in a rigid manner imitating a tool (e.g. the index finger in drawing and writing). In fact, a previous study (Kroliczak and Frey, G 2009) contrasting tool actions with communicative gestures (similar to direct communication) reported activation of similar parietal regions. Second, indirect communication, unlike actions of the other classes, may require continuous monitoring of peri-personal space to know when to terminate the action (Ghafouri and Lestienne, 2000; Brozzoli et al., 2010; Patane et al., 2020). This differential association of sensorimotor with spatial processing may be one driving force for the specificity of PFt activation. Finally, PFt may maintain the "mind's eye" of the trace to be left on the substrate in indirect communication actions, as has been postulated for planning artwork (Morriss-Kay, 2010). In other words, the actor needs to keep a mental image of the thing they will draw/write/sculpt while they are performing the action (e.g. a heart image while drawing a heart shape on the sand). The other classes do not require such a mental image: here external signals guide the transformation: size and orientation for manipulation (e.g. to know how to grasp, Culham and Valyear, 2006), or the height of the human target for direct communication (e.g. to know how much to raise the hand to wave). In addition to the difference in activation by the action classes, the specific involvement of PFt in processing observed indirect communication is further supported by the differential activation of PFt by the indirect communication exemplars, which was specific to the video conditions (Fig 7). While right DIPSA and DIPSM showed similar activity, the effects were larger and bilateral in PFt. However, the observed indirect communication exemplars also differed in the effector used, although all of them involved the distal upper limb. The absence of differential effects in PFt of the exemplars of the other action classes, which also differed in the effectors used, strongly suggest that the differential effect of the indirect communication exemplars reflected the action itself and not the effectors. 
OCCIPITO-TEMPORAL (MTG)



B.

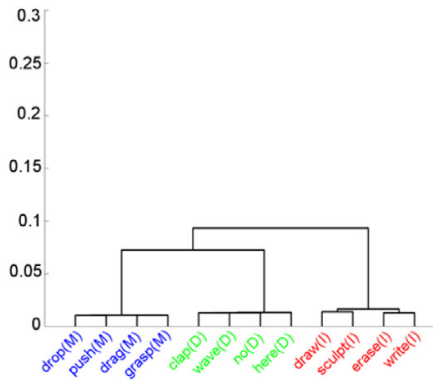

C.

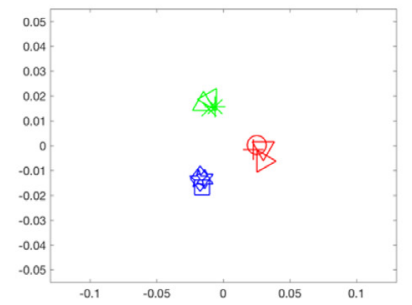

D.

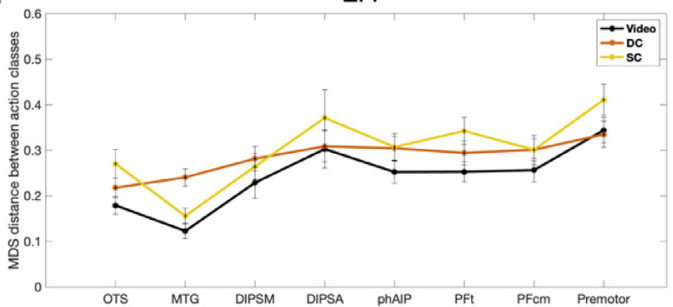

PARIETAL

(phAIP)
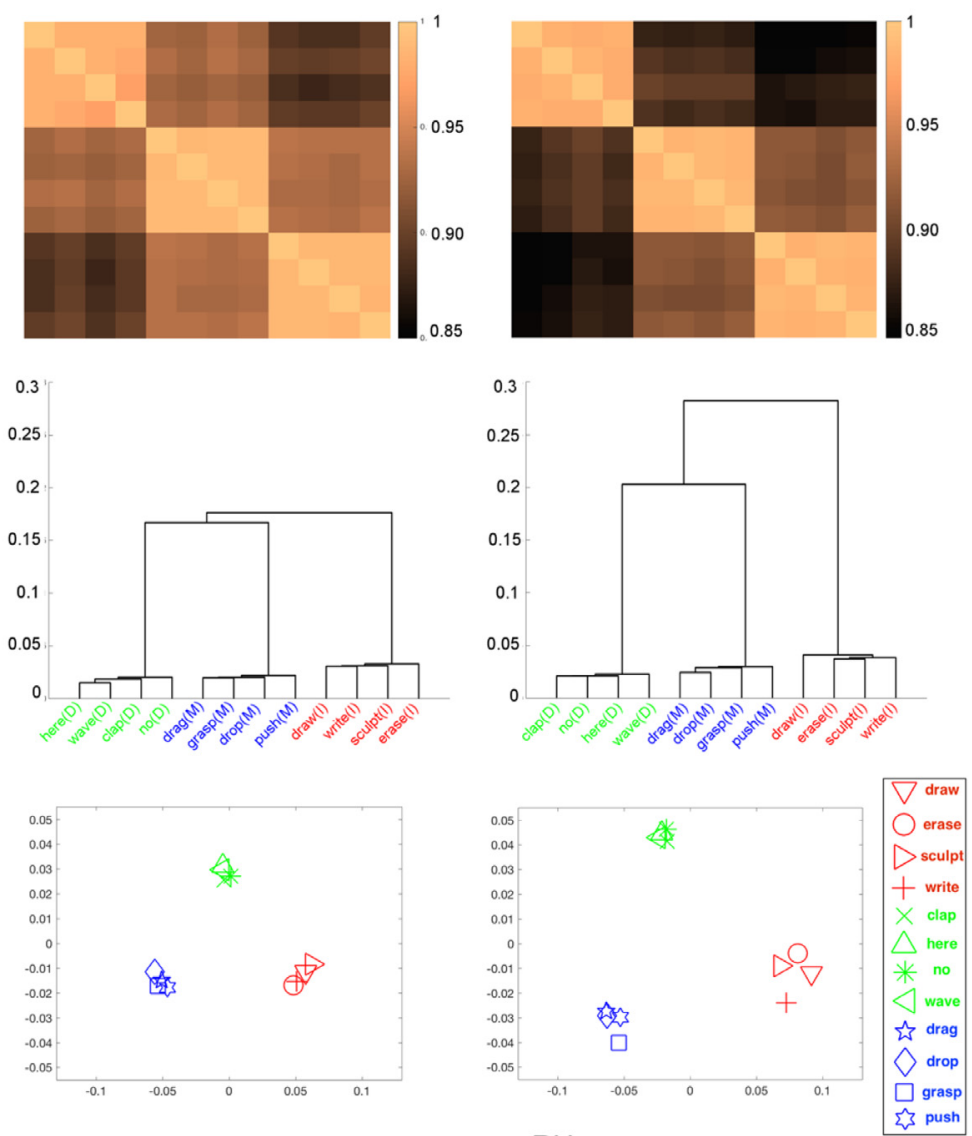

$\mathrm{RH}$

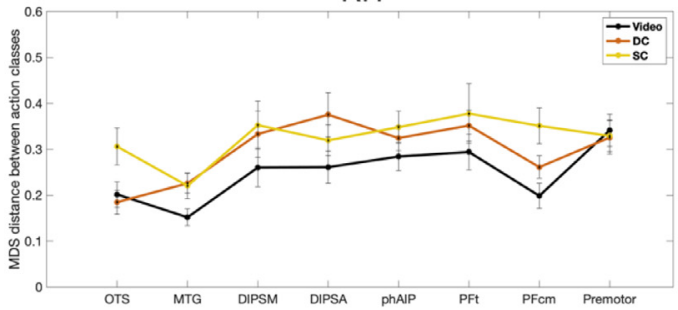



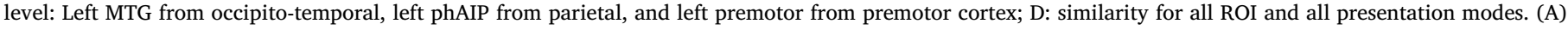

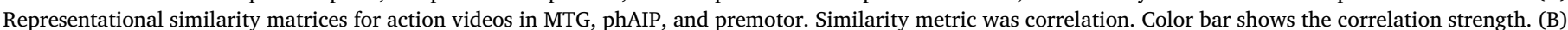

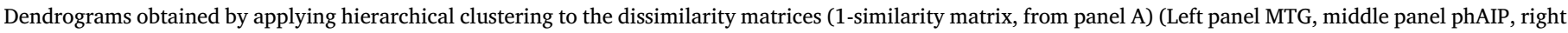

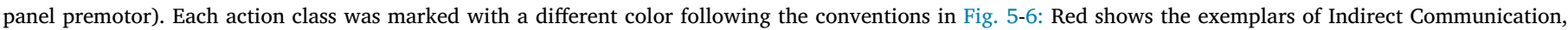

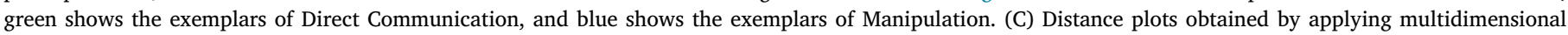

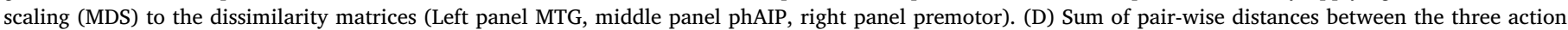

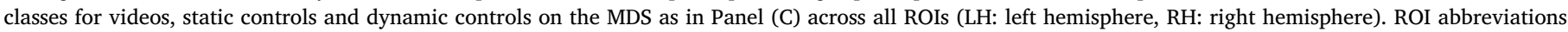

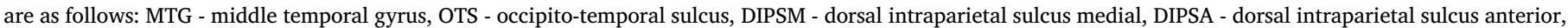
phAIP - putative human AIP.

Our results also qualify the role of phAIP whose involvement in observing manipulation is well established (Jastorff et al., 2010; Abdollahi et al., 2013; Ferri et al., 2015; 2016; Corbo and Orban, 2017; Orban et al., 2019; Aflalo et al., 2020). Bilateral phAIP was involved in manipulation and indirect communication actions, which correspond to relatively similar sensorimotor transformations as the hand interacts with either an object (with the goal of displacing or deforming) or a substrate (with the goal of transforming). In other words, indirect communication involves modifying the substrate, which is similar to manip- ulating objects - you cannot move the substrate but you deform it, and the sensory input for its planning is the 3D shape of the substrate and the 3D texture which are also used in manipulation. On the other hand, the absence of phAIP involvement in observing direct communication is reminiscent of the distinction between observing intransitive and transitive actions, the former failing to activate the rostral part of IPS relative to seeing a moving dot (Jonas et al., 2007). Instead, direct communication actions activated, area PFcm (non-specifically at the parietal level), 
an area which was previously shown to be activated by observed vocal communication actions (e.g. speaking, Corbo and Orban, 2017).

While our results show a specific site in parietal cortex for observed indirect communication actions, no such specific site was found for direct communication or manipulation actions. While an earlier study reported the overlapping parietal activation by viewing emblems and grasping to reflect the common effector (the hand, Andric et al., 2013), the additional control conditions in the present study suggest several alternative explanations for this absence of specificity. First of all, specificity of an action class is defined relative to the other action classes involved in the study. Hence, the similarity of action classes that are compared to each other is an important factor. As we discussed above, manipulation actions share similar motor goals with indirect communication actions, i.e. deforming an object or a substrate. However, on top of this goal, indirect communication actions require the actor to maintain a mental image of the thing that will be created. So, one could argue that indirect communication actions could recruit the similar neural resources as manipulation actions but would recruit additional resources for supporting the maintenance of a mental image. This implies that there would be no specific region for manipulation actions in the present study, contrary to earlier ones (Abdollahi et al., 2013; Ferri et al., 2015; Corbo and Orban 2017). A second explanation for the lack of clusters for direct communication and manipulation actions is that $\mathrm{PFcm}$, the region where we see the largest activation for direct communication actions (thus might expect to see a specific activation for direct communication) is a common region for all three action classes as evidenced by the common activation map (Fig. 4). Previous research shows on the one hand that manipulation and vocal communication might be segregated in PFcm along its dorso-ventral axis (Ferri et al., 2016; Corbo and Orban 2017, see Fig. 4), and on the other hand, that this region has a large anatomical variability across subjects (Hickok et al., 2009). Thus, it is possible that individual variability in responses to direct communication and manipulation actions hinder segregation and thus specific activations for these two action classes. Indeed, probing $\mathrm{PFcm}$ voxels with the specific activation contrast for direct communication yields a local maximum $(t=1.8$ at $p<0.1)$ in the most ventral part of PFcm.

Finally, our results suggest that what matters at the parietal level is the immediate goal of an action. We originally defined indirect and direct communication actions as communicative actions since they share the (distant) goal of communicating a message to a conspecific. However, the activation maps of direct and indirect communication actions do not show similar activity patterns at the level of the parietal cortex. Rather, indirect communication and manipulation actions, which share a common immediate goal (i.e. modifying a substrate or an object) show similar activity patterns. This suggests that at the level of the parietal cortex, what is coded is the immediate (observable) goal rather than the unobservable distant goal.

\subsection{Comparison of the three levels of the aon}

Our study reveals that the parietal level segregates the observed action classes more distinctly than the two other levels. Indeed, the specific map included larger activation sites at the parietal level than at the two other, while the common map was more extensive in the OTC than at the other two (Table 4). The parietal level also exhibited the greatest univariate separation (Figs. 5-6), and best discrimination between the videos of the three action classes (Fig. 8), an effect specific for the videos compared to controls.

Together, these findings shed light on the role of each level in action observation. This and previous studies suggest that the highest level of observed action identity, action class, is processed in anatomically segregated PPC regions, in all likelihood because action observation shared neural structures with action planning (Ferri et al., 2015). These structures, the "intentional maps" (Anderson and Buneo, RA 2002), implement the sensorimotor transformations used in the definition of action classes. That many of the class-specific PPC activation sites are located in rostral PPC is consistent with the findings of Roth and Zohary (2015) that position invariance increases along a caudo-rostral gradient in PPC.

If the occipito-temporal and premotor levels of the AON do not process observed action identity what, then, is their contribution? Given that the OTC sites are shared by many action classes, they may process factors common to these many different actions such as the actor (Orban 2015, Cross et al., 2009b), or more abstract factors such as agency (Sperduti et al., 2011). There is also evidence that OTC sites code some abstract aspects of observed actions such as category of target objects (Wurm and Lingnau, 2015), transitivity (Wurm et al., 2017), or sociality and interaction envelope (Tarhan and Konkle, 2020), which might result from using actions with man-made objects (e.g. tools, boxes) or complex scenes (e.g. using a knife in the kitchen), especially when presented as static stimuli. On the other hand, recent evidence suggests that the number of fingers used in grasping is encoded at the premotor level (Fabbri et al., 2016). This is consistent with the suggestion that in action observation the premotor cortex processes the effector of the action, because premotor, and not parietal cortex exhibits a somatotopic organization (Jastorff et al., 2010). This is supported by the lack of class segregation in the premotor cortex, because the effectors used in the three observed action classes were matched, and by the increase in representational distance between the exemplars, which differ in effectors, in premotor cortex compared to the other levels (Fig. 9C).

\subsection{Cross-method comparison between univariate analysis, MVPA, and $R S A$}

In the present study, univariate analysis and multivariate techniques were complementary, but differences in the results provided us some caveats in using these techniques.

First, we note that the specification of the spatial extent of an ROI is crucial. In the present study, the lack of differences between action classes in PFcm with univariate analysis as opposed to the above-chance performance in the MVPA could be explained by the distinct activation patterns in the dorsal and ventral PFcm for manipulation and communication actions. Earlier research with manipulation actions showed activation in the relatively more dorsal part of PFcm (Ferri et al., 2015) whereas earlier research with vocal communication actions showed activation in the relatively ventral part of PFcm (Corbo and Orban, 2017) (See Fig. 4B). These findings suggest that communication and manipulation actions were represented by distinct population responses in $\mathrm{PFcm}$ and that the univariate analysis might have missed that pattern information by averaging across voxels, which may also be the case for some studies that reported lack of difference between manipulation and communicative actions at the parietal cortex (Montgomery et al., 2007).

Second, the interpretation of MVPA results in fMRI experiments using complex stimuli such as natural images or videos may be difficult if control stimuli are not used. The videos as well as the static and dynamic controls showed above-chance performance. This raises the possibility that not only the videos, but also the controls, includes several features other than the action, some of them complex, which differed between classes and were apparently utilized by the decoding algorithms. This especially becomes a problem when the feature is linked to a single class, such as the conspecific on the left of the direct communication videos. Hence our results underscore the superiority of a classification of multiple items (and certainly more than two), and the desirability of decoding of control stimuli to verify the specificity of the classification, in line with caveats for interpreting fMRI MVPA results in terms of neuronal selectivity (Dubois et al., 2015).

The final point concerns the comparison of multivariate techniques, given the discrepancies between MVPA and RSA results: Although both methods showed separation between the three levels of the action observation network, the separation shown by MVPA was specific to video stimuli as opposed to the control stimuli whereas RSA separation was similar for all presentation modes. MVPA showed the greatest separa- 
tion at the parietal level. This discrepancy suggests that although both techniques use multivariate data, the different metrics reveal distinct aspects of the data, with discriminability (MVPA) being a more discrete measure (above-chance or not), and distance (RSA) more continuous. Furthermore, MVPA can detect subtle biases in voxels across conditions (Kamitani and Tong, 2005) to which RSA may be less sensitive. Regardless, the fact that MVPA shows distinctions between the three levels of the action observation network adds further evidence that the three levels process different aspects of the observed actions.

\subsection{Limitations}

One important limitation of the present study is the limited number of action classes that were compared to characterize the specific activation map of an action class. In the present study, we used two classes (e.g. direct communication and manipulation) to define the specific map of a particular action class (e.g. indirect communication). This was done following the conventions of previous work (Abdollahi et al., 2013; Ferri et al., 2015; Corbo and Orban, 2017) for methodological consistency. However, this approach limits one to define specificity relative to the action classes compared and to make generalizations. Future work could use a large number of action classes and exemplars in the human repertoire to better characterize specificity and anatomical segregation at PPC.

A second limitation is that the present study in its current form cannot distinguish between alternative theories for the contribution of area PFt in processing indirect communication actions. As explained in Section 4.1, indirect communication actions are distinguished from the other two classes in terms of featuring tool-like kinematics, the use of peri-personal space, and maintenance of a mental image of the thing that would be created. Future work could systematically manipulate these variables to disentangle these hypotheses for the contribution of area PFt.

Another limitation of the present study is that the primary effector used in all action classes was hand (as a whole or several fingers). It remains unknown whether the specific activation map for indirect communication depends on the effector that is used. Although the primary goal of the present study was not to study effector-dependency, future work should investigate whether specific maps especially at the level of parietal cortex are effector-invariant, not only for indirect communication actions but for many action classes in the human repertoire. We also believe that the lack of clear differences between the parietal and premotor levels of the AON as compared to the robust differences between occipito-temporal and parietal levels is due to the use of the same effectors in all action classes. So, varying the effector in search of specific maps with a larger set of action classes is a path that is worth of pursuing.

A final set of limitations is common to all fMRI experiments. The signal measured in fMRI studies is the hemodynamic response function which has a long time constant. This prevents the detection of very brief electrical events such as the short activation of anterior temporal lobe in gender discrimination in action perception (Platonov et al., 2019). Given the long duration of the videos and the matching duration of neuronal activation in human rostral IPS (Aflalo et al., 2020), this is not an issue in the present study. fMRI studies have, however, another limitation: the pooling of neuronal responses over large number of neurons. Single cell studies have shown that overlapping fMRI activations in two experimental conditions can correspond either to a common neural substrate for action verbs and observed actions (Aflalo et al., 2020) or to segregated substrates for attended locations and motor goals (Messinger et al., 2021). Thus, we do not know whether the overlapping activation of rostral IPS by indirect communication and manipulation classes indicates that neurons selective for manipulation exemplars (Aflalo et al., 2020) are also selective for indirect communication exemplars, or on the contrary that segregated neuronal population coding for manipulation and indirect communication exemplars. The differential effects of indirect communication exemplars, but not manipulation exemplars, in DIPSA favors the segregated hypothesis, but further single neuron studies are needed to address this issue.

\subsection{Conclusion}

In the present study, we extended previous work that shows anatomical segregation of posterior parietal cortex for different observed action classes with a new action class, namely indirect communication, whose exemplars include actions such as writing or drawing. We found a specific activation site in area PFt for indirect communication actions as compared to manipulation actions which share similar immediate goals, and direct communication actions, which share similar distant goals. In addition, we show that action class information can be decoded best at the parietal level of the AON as compared to the other two anatomical levels, namely occipito-temporal and premotor cortex. We hope that future work can extend this study by incorporating a larger set of actions covering the human repertoire.

\section{Data code availability}

All data and code used in the study are available upon request from the corresponding author.

\section{Credit authorship contribution statement}

Burcu A. Urgen: Conceptualization, Methodology, Software, Formal analysis, Investigation, Writing - original draft, Writing - review \& editing, Visualization. Guy A. Orban: Conceptualization, Methodology, Investigation, Writing - original draft, Writing - review \& editing, Supervision, Project administration, Funding acquisition.

\section{Acknowledgments}

This study was supported by ERC AdG-2012 323606 (Parietal action) to Guy A. Orban. The authors would like to thank to Stefania Ferri and Daniele Corbo for their help in preparation of stimuli and data collection. The authors declare no competing financial interests.

\section{References}

Abdollahi, R.O., Jastorff, J., Orban, G.A., 2013. Common and segregated processing of observed actions in human SPL. Cereb. Cortex 23 (11), 2734-2753.

Abdollahi, R.O., Kolster, H., Glasser, M.F., Robinson, E.C., Coalson, T.S., Dierker, D., Jenkinson, M., Van Essen, D.C., Orban, G.A., 2014. Correspondences between retinotopic areas and myelin maps in human visual cortex. Neuroimage 99, 509-524.

Aflalo, T., Zhang, C., Rosario, E., Pouratian, N., Orban, G.A., Andersen, R.A., 2020. A shared neural substrate for action verbs and observed actions in human posterior parietal cortex. Sci. Adv. 6 (43), eabb3984.

Andersen, R.A., Buneo, C.A., 2002. Intentional maps in posterior parietal cortex. Annu. Rev. Neurosci. 25, 189-220.

Andric, M., Solodkin, A., Buccino, G., Goldin-Meadow, S., Rizzolatti, G., Small, S.L., 2013. Brain function overlaps when people observe emblems, speech, and grasping. Neuropsychologia 51 (8), 1619-1629.

Beauchamp, M.S., Lee, K.E., Haxby, J.V., Martin, A., 2002. Parallel visual motion processing streams for manipulable objects and human movements. Neuron 34 (1), 149-159.

Brozzoli, C., Cardinali, L., Pavani, F., Farnè, A., 2010. Action-specific remapping of peripersonal space. Neuropsychologia 48 (3), 796-802.

Caspers, S., Geyer, S., Schleicher, A., Mohlberg, H., Amunts, K., Zilles, K., 2006. The human inferior parietal cortex: cytoarchitectonic parcellation and interindividual variability. Neuroimage 33 (2), 430-448.

Caspers, S., Zilles, K., Laird, A.R., Eickhoff, S.B., 2010. ALE meta-analysis of action observation and imitation in the human brain. Neuroimage 50 (3), 1148-1167.

Chan, A.W., Baker, C.I., 2015. Seeing is not feeling: posterior parietal but not somatosensory cortex engagement during touch observation. J. Neurosci. 35 (4), 1468-1480.

Chang, C., Lin, C., 2011. LIBSVM: a library for support vector machines. ACM Trans. Intell. Syst. Technol. 2 (27), 1-27:27.

Corbo, D., Orban, G.A., 2017. Observing others speak or sing activates Spt and neighboring parietal cortex. J. Cogn. Neurosci.

Cortes, A., Vapnik, V., 1995. Support-vector networks. Mach. Learn. 20 (3), 273-297.

Cross, E.S., Kraemer, D.J., Hamilton, A.F., Kelley, W.M., Grafton, S.T., 2009a. Sensitivity of the action observation network to physical and observational learning. Cereb. Cortex 19 (2), 315-326. 
Cross, E.S., Hamilton, A.F., Kraemer, D.J., Kelley, W.M., Grafton, S.T., 2009b. Dissociable substrates for body motion and physical experience in the human action observation network. Eur. J. Neurosci. 30 (7), 1383-1392.

Culham, J.C., Valyear, K.F., 2006. Human parietal cortex in action. Curr. Opin. Neurobiol. 16 (2), 205-212.

Di Dio, C., Di Cesare, G., Higuchi, S., Roberts, N., Vogt, S., Rizzolatti, G., 2013. The neural correlates of velocity processing during the observation of a biological effector in the parietal and premotor cortex. Neuroimage 64, 425-436.

Fabbri, S., Stubbs, K.M., Cusack, R., Culham, J.C., 2016. Disentangling representations of object and grasp properties in the human brain. Journal of Neuroscience 36 (29), 7648-76622.

Ferri, S., Rizzolatti, G., Orban, G.A., 2015. The organization of the posterior parietal cortex devoted to upper limb actions: an fMRI study. Hum. Brain Mapp. 36 (10), 3845-3866.

Ferri, S., Pauwels, K., Rizzolatti, G., Orban, G.A., 2016. Stereoscopically Observing Manipulative Actions. Cereb. Cortex 26 (8), 3591-3610.

Fujii, N., Hihara, S., Iriki, A., 2008. Social cognition in premotor and parietal cortex. Soc. Neurosci. 3 (3-4), 250-260.

Ghafouri, M., Lestienne, F.G., 2000. Altered representation of peripersonal space in the elderly human subject: a sensorimotor approach. Neurosci. Lett. 289 (3), 193-196.

Giese, M.A., Poggio, T., 2003. Neural mechanisms for the recognition of biological movements. Nat. Rev. Neurosci. 4 (3), 179-192.

Goodale M.A., Milner A.D. (1992) Separate visual pathways for perception and action. TINS, 15(1).

Graham, K.E., Hobaiter, C., Ounsley, J., Furuichi, T., Byrne, R.W., 2018. Bonobo and chimpanzee gestures overlap extensively in meaning. PLoS Biol. 16 (2), e2004825.

Graziano, M.S., Aflalo, T.N., 2007. Mapping behavioral repertoire onto the cortex. Neuron 56 (2), 239-251.

Holmes, A.P., Friston, K.J., 1998. Generalisability, random effects \& population inference. Neuroimage. In: 4th International Conference on Functional Mapping of the Human Brain, p. S754.

Hickok, G., Okada, K., Serences, J.T., 2009. Area Spt in the human planum temporale supports sensory-motor integration for speech processing. J. Neurophysiol. 101 (5), 2725-2732.

Jastorff, J., Orban, G.A., 2009. Human functional magnetic resonance imaging reveals separation and integration of shape and motion cues in biological motion processing. J. Neurosci. 29 (22), 7315-7329.

Jastorff, J., Begliomini, C., Fabbri-Destro, M., Rizzolatti, G., Orban, G.A., 2010. Coding observed motor acts: different organizational principles in the parietal and premotor cortex of humans. J. Neurophysiol. 104 (1), 128-140.

Jastorff, J., Popivanov, I.D., Vogels, R., Vanduffel, W., Orban, G.A., 2012. Integration of shape and motion cues in biological motion processing in the monkey STS. Neuroimage 60 (2), 911-921.

Jonas, M., Siebner, H.R., Biermann-Ruben, K., Kessler, K., Bäumer, T., Büchel, C., Schnitzler, A., Münchau, A., 2007. Do simple intransitive finger movements consistently activate frontoparietal mirror neuron areas in humans? Neuroimage 36 (2), T44-T53 Suppl.

Kaas, J.H., Stepniewska, I., 2016. Evolution of posterior parietal cortex and parietal-frontal networks for specific actions in primates. J. Comp. Neurol. 524 (3), 595-608.

Kamitani, Y., Tong, F., 2005. Decoding the visual and subjective contents of the human brain. Nat. Neurosci. 8 (5), 679-685.

Kriegeskorte, N., Mur, M., Bandettini, P., 2008. Representational similarity analysis - connecting the branches of systems neuroscience. Front. Syst. Neurosci. 2, 4.

Króliczak, G., Frey, S.H., 2009. A common network in the left cerebral hemisphere represents planning of tool use pantomimes and familiar intransitive gestures at the hand-independent level. Cereb. Cortex 19 (10), 2396-2410.

Liebal, K., Call, J., 2012. The origins of non-human primates' manual gestures. Philos. Trans. R. Soc. Lond. B Biol. Sci. 367 (1585), 118-128.

Liu, N., Kriegeskorte, N., Mur, M., Hadj-Bouziane, F., Luh, W.M., Tootell, R.B., Ungerleider, L.G., 2013. Intrinsic structure of visual exemplar and category representations in macaque brain. J. Neurosci. 33 (28), 11346-11360.
Messinger, A., Cirillo, R., Wise, S.P., Genovesio, A., 2021. Separable neuronal contributions to covertly attended locations and movement goals in macaque frontal cortex. Sci. Adv. 7 (14), eabe0716.

Montgomery, K.J., Isenberg, N., Haxby, J.V., 2007. Communicative hand gestures and object-directed hand movements activated the mirror neuron system. Soc. Cogn. Affect. Neurosci. 2 (2), 114-122.

Morriss-Kay, G.M., 2010. The evolution of human artistic creativity. J. Anat. 216 (2), 158-176.

Muller-Putz, G.R., Scherer, R., Brunner, C., Leeb, R., Pfurtscheller, G, 2008. Better than random: a closer look on $\mathrm{BCI}$ results. Int. J. Biomagn. 10, 52-55.

Nelissen, K., Borra, E., Gerbella, M., Rozzi, S., Luppino, G., Vanduffel, W., Rizzolatti, G., Orban, G.A., 2011. Action observation circuits in the macaque monkey cortex. J. Neurosci. 31 (10), 3743-3756.

Orban, G.A., 2016. Functional definitions of parietal areas in human and non-human primates. Proc. Biol. Sci. 283 (1828).

Orban, G.A., Lanzilotto, M., Bonini, L., 2021. From Observed Action Identity to Social Affordances. Trends Cogn. Sci. (Regul. Ed.).

Orban, G.A., Ferri, S., Platonov, A., 2019. The role of putative human anterior intraparietal sulcus area in observed manipulative action discrimination. Brain Behav. 9 (3), e01226.

Patané, I., Brozzoli, C., Koun, E., Frassinetti, F., Farnè, A., 2020. Me, you, and our object: peripersonal space recruitment during executed and observed actions depends on object ownership. J. Exp. Psychol. Advance online publication.

Peeters, R., Simone, L., Nelissen, K., Fabbri-Destro, M., Vanduffel, W., Rizzolatti, G., Orban, G.A., 2009. The representation of tool use in humans and monkeys: common and uniquely human features. J. Neurosci. 29 (37), 11523-11539.

Peeters, R.R., Rizzolatti, G., Orban, G.A., 2013. Functional properties of the left parietal tool use region. Neuroimage 78, 83-93.

Pereira, F., Mitchell, T., Botvinick, M., 2009. Machine learning classifiers and fMRI: a tutorial overview. Neuroimage 45 (1), S199-S209 Suppl.

Platonov, A., Avanzini, P., Pelliccia, V., LoRusso, G., Sartori, I., Orban, G.A., 2019. Rapid and specific processing of person-related information in human anterior temporal lobe. Commun. Biol. 2 (1), 1-9.

Rizzolatti, G., Craighero, L., 2004. The mirror-neuron system. Annu. Rev. Neurosci. 27, 169-192.

Roth, Z.N., Zohary, E., 2015. Position and Identity Information Available in fMRI Patterns of Activity in Human Visual Cortex. J. Neurosci. 35 (33), 11559-11571.

Sperduti, M., Delaveau, P., Fossati, P., Nadel, J., 2011. Different brain structures related to self- and external-agency attribution: a brief review and meta-analysis. Brain Struct. Funct. 216 (2), 151-157.

Tarhan, L., Konkle, T., 2020. Sociality and interaction envelope organize visual action representations. Nat. Commun. 11 (1), 1-11.

Urgen, B.A., Pehlivan, S., Saygin, A.P., 2019. Distinct representations in occipito-temporal, parietal and premotor cortex during action perception revealed by fMRI and computational modeling. Neuropsychologia 127, 35-47.

Van Essen, D.C., 2005. A Population-Average, Landmark- and Surface-based (PALS) atlas of human cerebral cortex. Neuroimage 28 (3), 635-662.

Van Essen, D.C., Drury, H.A., Dickson, J., Harwell, J., Hanlon, D., Anderson , C.H., 2001. An integrated software suite for surface-based analyses of cerebral cortex. J. Am. Med. Inform. Assoc. 8 (5), 443-459.

Van Essen, D.C., Dierker, D.L., 2007. Surface-based and probabilistic atlases of primate cerebral cortex. Neuron 56 (2), 209-225.

Vangeneugden, J., Pollick, F., Vogels, R., 2009. Functional differentiation of macaque visual temporal cortical neurons using a parametric action space. Cereb. Cortex 19 (3), 593-611.

Wurm, M.F., Lingnau, A., 2015. Decoding actions at different levels of abstraction. J. Neurosci. 35 (20), 7727-7735.

Wurm, M.F., Caramazza, A., Lingnau, A., 2017. Action categories in lateral occipitotemporal cortex are organized along sociality and transitivity. J. Neurosci. 37 (3), 562-575. 